\title{
ABSTRACTS
}

\section{CAGP-CCSMH Annual Scientific Meeting Book Of Abstracts Building Resilience In Seniors' Mental Health In Canada October 25-26, 2019, Banff Alberta}

DOI:https://doi.org/10.5770/cgj.23.415

\section{Medical Assistance In Dying \\ Michael Trew}

Background: This workshop will review the history of MAID in Canada and around the world and then focus on the current requirements for eligibility. The role of psychiatry and mental health is twofold; to support people in their considerations for end-of-life planning, and at times to formally consider whether the person has capacity to proceed with MAID.

National Guidelines for Substance Use Disorders among Older Canadians: From Evidence to Action!

David Conn, Kiran Rabheru, Dallas Seitz, Chris Kitamura, Nancy Vasil, Lisa Van Bussel, Keri-Leigh Cassidy

Background: The Canadian Coalition for Seniors Mental Health has been committed to promoting the mental health of seniors by connecting people, ideas and resources. Substance use disorders and substance misuse among older adults are a common yet neglected issue. In the summer of 2017, the CCSMH embarked on a 3-year project leading and facilitating the development of clinical guidelines and knowledge translation tools addressing Alcohol, Cannabis, Benzodiazepine and Opioid use disorders among seniors. Resulting Clinical Guidelines will be published this year with communityoriented tools being finalized in 2019-2020.

Method: The CCSMH has been funded by Health Canada to produce national clinical guidelines for substance use disorders among older adults. Working groups with members from across Canada and from diverse disciplines (including geriatric psychiatry and medicine, addictions medicine, pharmacy, family medicine, psychology, social work, nursing, policy and people with lived experience) have dedicated the past two years to addressing the unique challenges of developing clinical guidelines and creating key knowledge translation products for clinicians, patients and family members.

Results: The Clinical Guidelines will be formally released in Summer 2019 and will include Low Risk Drinking Guidelines for older adults, recommendations regarding the treatment of opioid use disorder in the context of chronic pain management among older adults, calls for an overall reduction in the prescription of benzodiazepines, and descriptions of the clinicians' role in assessing cannabis use among older adults in light of new legislation and increasing use of "medical cannabis".

Conclusions: With the guidelines in their final stage of completion, CCSMH is now focused on moving from evidence to action through broad dissemination and extensive knowledge translation (KT) activities. Our KT product portfolio will include: clinical pocket cards, presentations and webinars for healthcare professionals, as well as brochures and presentations designed for older adults, their families and caregivers.

Innovative Approaches to Managing Acute and Protracted Withdrawal from Alcohol and Sedative-Hypnotics in Seniors

Peter Chan, Ashok Krishnamoorthy

Background: Alcohol and sedative-hypnotic use disorders in seniors can contribute to falls, fractures, malnutrition, self-neglect, and neurocognitive disorder. Withdrawing from alcohol or sedative-hypnotics can be often challenging, particularly in those who are frail, have multiple comorbid medical conditions, or have a longstanding psychiatric disorder. Recent publications have highlighted some approaches to managing addiction and withdrawal, such as the Canadian Coalition of Seniors' Mental Health's (CCSMH) Practice Guidelines for Substance Use Disorders (2019) and the Cochrane review for pharmacological management of benzodiazepine withdrawal (2018). 


\section{CAGP-CCSMH ABSTRACTS}

Method: Two retrospective studies of a CIWA-based, symptom-triggered detoxification protocol employed since 2010 at a teaching hospital's elder care units will be presented. These studies will be compared to other symptom-triggered and fixed dose studies. Also, a successful case of withdrawing a hospitalized elderly benzodiazepine-dependent patient with the aid of valproic acid will be presented. We will also present an evidence-informed community approach for managing protracted alcohol withdrawal and beyond.

Results: A CIWA-based, symptom-triggered approach demonstrated decreased total duration of benzodiazepine use, cumulative benzodiazepine dose, and the use of adjunctive sedative medications. The approach can be combined with prophylactic anticonvulsants to prevent more severe alcohol withdrawal symptoms from emerging. The use of anti-craving agents such as acamprosate, naltrexone, and gabapentin in the protracted phase of alcohol withdrawal can help maintain long-term abstinence.

Conclusions: There is a role for anticonvulsants and symptom-triggered protocols in the management of alcohol or sedative-hypnotic withdrawal. An integrated model of community care for alcohol use disorder can be effective in maintaining sobriety.

\section{Nothing About Us Without Us: Creating the First Canad- ian Charter of Rights for People with Dementia}

Riley Malvern, Mary Schulz, Christene Gordon

Background: People with dementia strongly advocate for their inclusion in the decisions that affect them, but often feel excluded from these conversations because of the stigma surrounding the disease. Consequently, the Alzheimer Society of Canada (ASC) invited people with dementia to create an Advisory Group to inform its work. In 2018, ASC's Advisory Group led the development of the first Canadian Charter of Rights for People with Dementia.

Method: The groundwork for the Charter was laid through a combination of: lived experience of people with dementia; an environmental scan that built on the work of disability networks and included international approaches, relevant human rights legislation and an understanding of how a rights-based approach may work in a Canadian context; and existing ASC resources on the meaningful engagement of people with dementia.

Results: The first ever Canadian Charter of Rights for People with Dementia was created by people with dementia for people with dementia and the organizations that support them. The Charter identifies the human rights of people with dementia that are often overlooked. It can be used by those with lived experience to claim these rights and reinforce that they, too, can live well. By supporting the community participation of people with dementia, the Charter strengthens protective factors for their resilience.

Conclusions: The Charter is a living document that ASC's Advisory Group will continue to revise based on feedback from stakeholders. They will ensure that it continues to speak to the rights of all people with dementia, regardless of stage or circumstance.

\section{Acceptability of Personal Contact and Group Activity Inter- ventions to Address Loneliness for People with Dementia}

Hannah O'Rourke, Souraya Sidani, Brittany Walsh, Judy Prestwich, Haydn McLean, Nicole Jeffery, Sheylenne Pyde

Background: Loneliness is painful, predicts depression, and older adults are at high risk. The purpose of this study was to assess acceptability of personal contact (human or animal) and group activity interventions to address loneliness for people with dementia.

Method: This was a mixed methods (concurrent triangulation) design using a convenience sample of family, friends, and health care providers of people with dementia $(n=25)$. In face-to-face sessions, participants were read a short description of each intervention and completed questionnaires to rate acceptability (adapted Treatment Perception and Preference measure). Semi-structured interviews $(\mathrm{n}=25)$ were used to explore perspectives in more depth. Descriptive statistics and conventional content analysis were completed.

Results: All three interventions were rated as effective, logical, suitable, and low risk by at least $80 \%$ of participants. Participants described how the interventions would address loneliness, but that they should be individualized to the person or the stage of dementia. Just $36 \%$ of participants rated group activity interventions as easy to deliver. This was related to participants' difficulty envisioning interaction between people with dementia, and highlighted that appropriate facilitation was essential. Willingness to participate ranged from $44 \%$ (for group activity) to $72 \%$ (for both personal contact interventions), and was influenced by time, scheduling concerns, and perceptions of roles and of the needs and abilities of people with dementia.

Conclusions: All three types of interventions appear promising and low risk. Future phase 2 trials should focus on assessing feasibility and acceptability of flexible personal contact and group activity interventions, and on the experiences of people with dementia.

\section{Person-Centred Language Matters!}

Tina Kalviainen, Kate Ducak 
Background: Behavioural Supports Ontario (BSO) wished to critically reflect and provide recommendations regarding language and communications used by BSO and its key stakeholders with the goal of promoting the consistent use of person-centred language that is appropriate, respectful, life-affirming and inclusive.

Method: BSO assembled an Expert Panel with key stakeholders from across Ontario to discuss language and its impacts. Deliberations resulted in a living document, including four BSO Person-Centred Language (PCL) Commitment Statements, informed by the authentic voice of persons with lived experience. Presentations were made to BSO's Provincial advisories to showcase the work and receive feedback.

Results: A report was released in October 2018. Translation tools developed to support the initiative included an online pledge platform, downloadable posters of the commitment statements and practical resources (www.behaviouralsupportsontario.ca/Pledge). A sold-out national webinar took place in early 2019, receiving endorsement by way of active participation and positive evaluations.

Conclusions: PCL can assist in establishing trusting relationships and removing barriers associated with fear and stigma. It can have a positive impact on how individuals feel, are viewed and are treated (as per Goddu and colleagues' 2018 article Do Words Matter? Stigmatizing Language and the Transmission of Bias in the Medical Record). BSO's work aligns with The Fountain of Health Initiative, emphasizing self-determination can be influenced by health care professionals. We can enhance outcomes by engaging in meaningful exchanges that include acknowledging and discussing dementia, complex mental health, substance use and/other neurological conditions, all of which are included in BSO's mandate and this PCL initiative.

\section{Advocating for Mental Health Services for Older Adults in Canada: Tools and Strategies}

Marie-France Tourigny-Rivard, Adele Loncar, Alexa Bol, Michèle Tremblay, Francine Knoops

Background: The Mental Health Commission of Canada (MHCC) published Guidelines for Comprehensive Mental Health Services for older Adults in Canada in 2011. Five years later, the MHCC wanted to develop knowledge translation activities to support the application of these guidelines.

Method: The MHCC held both telephone and in-person consultations with different organizations representing seniors, health care providers and planners, academic institutions and advocacy groups in regards to the use of these guidelines. This led the MHCC to develop three companion tools to facilitate the implementation of the recommendations contained in these guidelines.

Results: These tools are:

- a summary version of the Guidelines

- infographics to help make the case for investing mental health services for older adults in Canada and

- a Compendium of Geriatric Mental Health Services, which describes a range of exemplary programs and practices in geriatric mental health that have been developed in different provinces and nationally over the last 10 years. It includes details about resourcing and evaluation results to specifically help health care planners who may be considering the implementation of similar services.

Conclusions: This workshop will not only present these tools but will engage participants in developing specific plans to use these tools to support their current clinical activities and advocacy efforts.

\section{CBT Express: Using the Wellness App for Health Behavior Change and Resilience}

\section{Beverley Cassidy, Kiran Rabheru}

Background: Activating health behavior can reduce the risk of dementia and mitigate the negative impact of frailty on functioning. The Wellness App is a practical health behavior change tool developed by The Fountain of Health. The app allows users to set health goals and receive audio encouragement (CBT, ACT and positive psychology strategies) to support health behavior change. Target goal areas include physical activity to optimize mobility, social connection to reduce isolation and depression risk, and brain challenge goals to optimize cognitive function and mental health.

Method: The Wellness App prototype was piloted in frontline care in Nova Scotia and uses SMART goal setting with audio encouragements based on CBT, ACT and positive psychology strategies to support health goal adherence over 4-8 week periods. The Wellness App, along with Fountain of Health paper tools, is currently being trialled in a large scale (n-650) quality assurance study with CCSMH in frontline care settings nationally.

Results: Pilot data have found that users of The Wellness App were able to achieve their health goals (partially, completely or even exceed) greater than $80 \%$ of the time. The Wellness App was found to be easy to use as an intra office tool by $>80 \%$ of frontline clinicians.

Conclusions: The Wellness App is a versatile clinical tool that can be incorporated into daily care of patients to optimize health in key areas such as mobility, social isolation, mood regulation and cognitive function. 


\section{Reducing Harm Related to High Risk Behaviours Among Formerly Homeless Older Adults: A Calgary-based Quali- tative Case Study}

\section{Lara Nixon, Victoria Burns}

Background: Alarming numbers of older Canadians are experiencing co-occurring problematic substance use and other mental health challenges, poverty and homelessness. Few supportive housing models exist to suitably care for this diverse population with complex health and social needs. Calls have been made for improved access to harm reduction for older adults. Although alternative permanent supportive housing (PSH) models employing a harm reduction approach are widely being embraced across Canada and the United States, few target the unique needs of older people and staff and older adult resident perceptions are largely absent from the literature.

Method: Drawing on Rhode's Risk Environment framework, a constructivist grounded theory approach, and interviews with residents (6) and their service providers (9), this qualitative case study explored how harm reduction was perceived and experienced in a single-site PSH complex in Calgary, Canada which provides wrap-around multi-disciplinary housing, health, and social supports grounded in harm reduction philosophy.

Results: The analyses revealed that harm reduction was perceived and experienced through the overarching category of "meeting people where they're at". Enacting harm reduction was facilitated through interpersonal respect, and shifting focus of harm reduction efforts away from relying solely on individual level behaviour change. An unexpected consequence of enacting harm reduction was community building. Constraints on enacting harm reduction were also identified related to the physical, social and political environment.

Conclusions: This study provides evidence to inform health and housing policies and programming targeting older vulnerable populations.

\section{An Immersive Simulation to Build Empathy for Geriatric} Patients with Co-occurring Physical and Mental Illness

Petal Abdool, Anika Saiva, Sarah Colman, Laura Hayos, Laura Naismith, Shaheen A. Darani, Latika Nirula

Background: As we face the growing aging population, it is imperative for health professionals to gain proficiency in working with elderly patients who suffer from co-occurring physical and mental illness. A key challenge lies in developing and supporting continued empathy towards this stigmatized population. Simulation can provide standardized experiential learning opportunities to address this critical gap.
Method: The simulation design involves participants first taking on the perspective of a geriatric patient with chronic mental illness through a narrative writing exercise and then physically inhabiting this role by wearing an aging simulation suit to perform a medication management task. Participants then engage in a facilitated debriefing session and a narrative writing exercise. Interviews conducted 3 months post-intervention will explore the impact of the simulation experience on their clinical practice.

Results: The study is in the data analysis phase. Preliminary findings indicate that $93 \%$ of the participants experienced increased in empathy for geriatric patients following the immersive simulation. Additionally, $93.3 \%$ of the participants expressed their intent to change practice as an outcome of this experience. Responses to how they intend to change practice include:

"Inquire about physical limitations to treatment ... Try to validate experiences of difficulties rather than get frustrated."

"... Allow more humanity in interactions."

Participants also appreciated the immersive and interactive learning modality of simulation. Quotes include:

"A quick direct way to understand patient perspectives. Unique way of learning empathy I've never gotten anywhere else..."

"I liked how real it felt. Immediately in the physical and mental state of a geriatric patient"

Conclusions: The preliminary results are promising. We anticipate the simulation will result in increased empathy for geriatric patients and foster meaningful discussion regarding the challenges faced by this vulnerable population in the healthcare system.

Waterloo Wellington Behaviour Support Team (BSOT) Successful Transitions for Older Adults with Opiate Use Disorders: A Case Study

Audrey Devitt, Marilyn White Campbell, Meldon Kahan, Tiffany Symes, Matilda Kress

Background: Older Adults with Substance Use disorders (SUD) are often perceived as a challenge to accommodate in long term care. The complexities of substance use in late life can be a barrier to accessing long term care beds. The suggestion of a substance use problem and the accompanying stigma associated with addictions can raise concerns for homes who feel ill equipped to provide care. Older Adults are among the highest users of Opiates and have the highest rates of hospitalizations with longer than average length of hospital stays than in any other age group. Through an exploration of this case study we will demonstrate the pathways and treatment that lead to successful transitions. 
Method: Condensed Case study involving detailed examination of critical incidents' and Emergency Department visits identifying the context and conditions of the patient and their outcome. A brief video of a person with Lived Experience will be featured.

Results: Using integrated collaborative approaches including the BSO pillars of System Coordination, interdisciplinary service delivery and knowledgeable teams and capacity building, the Waterloo Wellington Geriatric Systems/ St Joseph's Health Center Guelph / Women's College Hospital / the Centre for Addiction \& Mental Health developed partnerships to support older adults with Substance Use Disorders to make healthy transitions.

Conclusions: Older adults with Opiate Use Disorders can be successfully supported to transition to community, and/ or retirement home living. In this instance, with support from the Waterloo Wellington Behaviour Support Ontario Team an interdisciplinary team coordinated care service delivery approach, and knowledgeable care teams, the patient avoided admission to LTCH.

Electroconvulsive Therapy in Canada: Emerging Trends in Geriatric Practice: Part 1

Kiran Rabheru, Peter Chan, Caroline Gosselin, Robyn Waxman

Background: There is large global variation in electroconvulsive therapy (ECT) utilization, administration, and practice. Canada's comprehensive ECT survey has estimated that between 7000-8000 patients received approximately 67,000 treatments annually. The CANECTS group has identified gaps in practice and training standards (www.canects.org) and has created national guidelines to provide a framework for ECT clinics and for conducting audits. The goals for this work included effective education for clinicians, standardization of ECT practice, and enhancing patient / family user experience.

Method: This first of a two-part workshop is dedicated to discussing emerging Canadian trends for ECT in special clinical situations in older adults. Topics 1:

1. Management of breakthrough depression during a course of maintenance ECT (MECT) -K. Rabheru

2. Anesthesia and perioperative care for elderly patients with high seizure thresholds and for those with postictal agitation. - C. Gosselin

3. ECT for treating BPSD and agitation in dementia. Canadian multicenter data for ECT and BPSD will be presented- $\mathrm{P}$. Chan \& R. Waxman
Results: Improved knowledge transfer, better quality of care in ECT practice, and improved patient safety. Active audience participation leading to the sharing of ECT experience and knowledge will be strongly encouraged in both workshops.

Conclusions: The two workshops will lead to a higher level of skills, knowledge and confidence in the clinical practice, education, research, program evaluation, developing policy in the field of ECT.

\section{Integrating CBT for Insomnia (CBTi) Into Your Practice}

Christopher Kitamura, Mark Rapoport

Background: Chronic insomnia is a common and challenging clinical syndrome in older adults. Insomnia can present in the context of psychiatric and medical comorbidity, or be the presenting issue in and of itself. Cognitive behavioural therapy for insomnia (CBTi) is a first line treatment for chronic insomnia and arises from the behavioural model of insomnia.

Method: In this interactive workshop, participants will learn the core CBTi interventions including stimulus control therapy, sleep restriction therapy and sleep hygiene, and be aware of adjunctive treatments including deprescribing, cognitive therapies, relaxation training and mindfulness. Participants will apply these to a common clinical case of benzodiazepine dependence. We will review evidence for CBTi, including some of our findings. We will review innovative treatment models (eg. single session, telemedicine, group, online, as well as CBTi for patients with cognitive impairment).

Results: By the end of the workshop, participants will learn a practical approach to integrating $\mathrm{CBTi}$ into their practice, be able to use CBTi to treat insomnia with benzodiazepine dependence, know the evidence for $\mathrm{CBTi}$, and be aware of innovative treatment models.

Conclusions: CBTi is an evidence-based treatment that should be integrated into regular practice.

The Behavioural Supports Ontario-Dementia Observation System (BSO-DOS $\left.{ }^{\circ}\right)$ : Interdisciplinary Innovation \& Collaboration Leading to Objective and Measurable Direct Observation Documentation

Debbie Hewitt Colborne, Lori Schindel Martin, Andrea Iaboni, Lisa Van Bussel, Adriana Barel, Julia Baxter, Monica Bretzlaff, Adriana Caggiano, Lina DeMattia, Gail Elliot, Fernanda Fresco, Katrina Grant, Pam Hamilton, Stephanie Jarvis, Teresa Judd, Lindy Kilik, Jodi Laking, Cecelia Marshall, Kristine Newman, Brynn Roberts, Kimberly Schlegel

Background: The care needs of people living with dementia experiencing responsive behaviours/personal expressions 


\section{CAGP-CCSMH ABSTRACTS}

are best determined when reliable data from a direct observational tool are considered in the assessment and planning process. Tailored care can then be based upon assessment of behavioural data reflecting the frequency, duration, level of risk and patterns of behaviour. Clinical teams need evidencebased measures to systematically record observed behaviours, identify contributing factors, tailor interventions and track outcomes. One such tool, the Dementia Observation System (DOS), developed in 1998, is widely used however there is no single version.

Method: In early 2017, Behavioural Supports Ontario (BSO) entered into an interprofessional collaboration with key stakeholders from across the Ontario to address this inconsistency. A provincial interdisciplinary DOS Working Group collected and reviewed over 40 versions of the DOS, identifying critical elements to create a new standardized version. Throughout the creation process, multiple stakeholders (including participants from the 2017 CAGP Conference) provided input and shaped the new standardized DOS. The new tool was trialed in the spring of 2018 to confirm its acceptability and feasibility.

Results: Based on this collaborative work, the new standardized BSO-DOS ${ }^{\odot}$ was finalized and released in March 2019. Several knowledge translation supports accompanied its release in order to foster meaningful utilization of the new BSO-DOS ${ }^{\circ}$.

Conclusions: The collaborative and inclusive approaches of the DOS Working Group will demonstrate that the BSO-DOS ${ }^{\circ}$ is evidence informed, draws on provincial/national innovations and is clinically relevant to multidisciplinary teams in their provision of person and family-centred care.

\section{Project ECHO: Utilizing Virtual Education to Build Cap- acity in Geriatric Mental Health}

David Conn, Lisa Sokoloff, Cindy Grief, Navena Lingum, Shaen Gingrich, Sid Feldman, Andrea Moser, James Chau, Salma Shaikh

Background: Project ECHO (Extension for Community Healthcare Outcomes) is a novel education program using videoconferencing technology to build capacity of primary care providers (PCPs), share knowledge through collaborative learning and discuss cases in real-time. ECHO facilitates Communities of Practice using a hub-and-spoke model, connecting specialists at academic centres (Hub) with PCPs (Spokes) particularly in rural, underserved areas. Originating in New Mexico, ECHO now reaches 34 counties. In 2018, Baycrest, collaborating with North East Specialized Geriatric Centre, launched ECHO Care of the Elderly (COE), the first ECHO in Canada focused on geriatric mental health and frail, complex aging patients.
Method: ECHO COE runs weekly in 10-15 week cycles. Each weekly session includes a short didactic presentation on a specific topic by the Hub team followed by cases presented by spokes. Questions, discussion and recommendations are skillfully facilitated by trained Hub members. Spokes have access to a website with resources, discussion board and recorded didactic presentations. During the workshop, a live mini "mock" ECHO session will be utilized to demonstrate.

Results: ECHO COE PCP participants have shown improvements in knowledge and self-efficacy. BPSD, mood disorders, dementia, sleep disorders and delirium were noted as being useful and relevant to participants' practice, indicating a need for knowledge sharing in geriatric mental health.

Conclusions: This workshop will describe the Project ECHO philosophy and methodology and identify the benefits of implementing an ECHO program for building capacity of PCPs in geriatric mental health. This workshop will provide participants with the simulated experience of collaborative learning through the $\mathrm{ECHO}$ process.

Beyond Dementia: An Interdisciplinary Approach to Managing Serious Mental Illness in Long-Term Care

Aviva Rostas, Leslie Giddens-Zuker

Background: Individuals with non-dementia mental illnesses such as depression, bipolar disorder and schizophrenia, are aging, and increasingly residing in the long-term care setting. These residents often differ from the "typical" long-term care resident in that they are often younger, less functionally impaired, and have varying support needs.

Method: A literature review was conducted with a focus on serious mental illness in the long-term care setting. During this presentation, an interactive discussion format will be used to review cases and explore unique clinical issues. Clinical tools will be introduced to aid in assessment.

Results: Residents with serious mental illness living in longterm care pose unique challenges to staff, who are more experienced in managing clients with dementia and associated behavioural and psychological symptoms. One important challenge is that there are often limited, if any, psychiatric records available. In terms of assessment, it can be difficult to determine whether a change in mental status relates to a primary mental illness, dementia, or an acute medical issue. From a treatment perspective, these residents are often on numerous psychotropic and potentially harmful medications. Additionally, there are issues around having these complex residents cohabiting in the same, at times limited, space, as vulnerable residents with dementia. We will also explore the use of mental health legislation, code whites, and stigma. 
Conclusions: We propose that an interdisciplinary model of care is critical to effectively meet the needs of this unique population, reduce unnecessary hospitalizations, and ensure safety of residents and staff in long-term care.

Paradoxical Complete Resolution of Depression, Psychosis, and Suicidal Ideation Following a Traumatic Brain Injury

Anthony Yeung, Mark Rapoport

Background: We describe the first case, to our knowledge, of a 68-year-old patient with complete resolution of multiple psychiatric symptoms (depression, psychosis, and suicidal ideation) immediately following a closed-head traumatic brain injury (TBI) involving the left frontal and temporal lobes. The patient's medications were bisoprolol $1.25 \mathrm{mg}$ PO daily, metformin 500mg PO BID (HbA1c 7.4\%), and multivitamins. He had no family history of psychiatric or neurodegenerative disorders. He was diagnosed with an index episode of depression by his family physician in September 2017. Several months later, he had a serious suicide attempt and was struck by a vehicle as a pedestrian in the middle of a highway.

Method: A non-contrast CT head revealed a subgaleal hematoma and left fronto-parieto-occipital subdural hematoma with minimal mass-effect and midline shift. He was immediately admitted to an intensive care unit for stabilization and observation. Over the following week, his intracranial injuries stabilized with conservative management. An MRI brain demonstrated the same left-sided hematoma with a mild local mass effect with no shift or herniation. Other findings on the MRI report included mild atrophy and white matter changes consistent with age.

Results: Remarkably, from the immediate aftermath of the accident to now 12-months post-recovery, the patient continues to have no psychiatric symptoms, and has returned to his baseline level of function.

Conclusions: The current neuropsychiatric literature largely focuses on the development of psychiatric disorders or behavioural symptoms as a post-injury sequalae of TBIs. We hope that our unique case report will spark further discussion on whether TBIs must necessarily cause unfavourable symptoms. In rare cases, it may even fortuitously resolve symptoms. A review of neuroanatomy and neurocircuitry may also help clinicians understand the pathophysiology behind common neuropsychiatric symptoms and disorders.

Post-Traumatic Stress Disorder in Major Neurocognitive Disorder: A Case Series

Marie-Andrée Bruneau, Klara Pokrzywko
Background: In late life, traumas may act cumulatively to exacerbate vulnerability to Post-Traumatic Stress Disorder (PTSD). PTSD is also a risk factor for cognitive decline. Major Neurocognitive disorder (MND) can be associated with worsening of already controlled PTSD symptoms, late-life resurgence or a de novo emergence. Misidentifying PTSD symptoms in MND can be of negative consequence for the patient and families.

Method: Our interactive presentation will include a brief introduction about the neurobiological and psychosocial interconnections between PTSD and MND. We will present 6 cases referred for consultation in geriatric psychiatry at the Institut universitaire de gériatrie de Montréal, initially for Behavioral and Psychological symptoms of Dementia (BPSD) which were eventually diagnosed and treated as PTSD.

Results: We propose that certain PTSD symptoms in patients with MND, are misinterpreted as BPSD, and therefore, not addressed properly. For example, flashbacks could be interpreted as hallucinations, hypervigilance as paranoia, nightmares as sleep disturbances and hyperreactivity as agitation/aggression. We suggest that better identification of PTSD symptoms in MND is needed. We also propose specific recommendations for care namely: gathering the most detailed history of trauma as possible, identifying potential triggers, modifying exposure psychotherapy, using antidepressants as first-line medications instead of benzodiazepines or antipsychotic medications, which are contraindicated in this population, and managing comorbidities.

Conclusions: Proper identification of PTSD symptoms in patients with MND will allow a more tailored and efficient treatment, with decrease in inappropriate use of physical and chemical restraints, decreased patient's distress and increased quality of life.

Integrated Mental Health and Medical Care in Action: Reports from the Frontlines of Clinical, System Planning, Leadership and Advocacy Efforts

Sophiya Benjamin

In 2018, the Canadian Academy of Geriatric Psychiatry promoted Integrated Mental Health and Medical Care as the annual theme. Catalyzed by such advocacy, there has been significant work done in the past year to close the gaps between medical and mental health providers and systems.

Clinical: The first section will describe the development, scale and spread of an innovative digital solution from a local pilot project to a provincial program. This model of care integrates geriatric psychiatry and geriatric medicine along with pharmacy and clinical pharmacology to provide access to geriatric expertise for seniors in Ontario irrespective of geographical location. 


\section{CAGP-CCSMH ABSTRACTS}

System Planning: The second part of the symposium will highlight a project that involved the mapping of specialized geriatric programs across Ontario including care provided by geriatric mental health and geriatric medicine. We will discuss opportunities and barriers encountered both in the process of the mapping as well as within the individual programs mapped.

Leadership: We will present a model in which an integrated physician leadership with representation from both psychiatry and medicine can foster understanding and collaboration between specialties. We will reflect on and engage in a discussion about the potentials and pitfalls of such an approach.

Advocacy: This section will describe the role of such integration in the structuring of governance models that can enable clinicians to organize and advocate for frail older adults.

The symposium will draw from the experiences of different experts doing such work in integration to stimulate dialogue and advocacy towards more integrated care.

Improved System Integration and Collaboration from Joint Leadership in Mental Health and Medical Care

Kevin Young

Background: In 2015 North Simcoe Muskoka, a region in Ontario with more seniors than the provincial average, was faced with the loss of several geriatric specialist practitioners due to lack of resources. A strategy was developed that led to the appointment of a tertiary care mental health hospital as the lead agency for specialized geriatric services.

Methods: With the development of a regional office and the integration of mental health and medical care teams there now exists leaders on the senior team including a Geriatric Psychiatrist, Geriatrician and administrators from both programs.

Results: Integrated leadership has led to the development of a common assessment tool that will allow mental heath and medical care teams to collaborate more easily. A joint annual education series for primary care physicians has been created. This and the creation of a central intake office has underscored to the primary care practitioners that we operate as one service and has led to efficiencies in system navigation. There is improved informal communication between specialists, and we continue to work toward offering interdisciplinary clinics. The integrated leadership model has been recognized at the provincial level and catalyzed discussions about the important of provincial integrated models of leadership.

Conclusions: We have demonstrated that integrated leadership reduces duplication of services and leads to alignment of patient care teams. We anticipate with ongoing collaboration patients will more successfully navigate the health care system.
Developing an Integrated Picture of Specialized Geriatric Services in Ontario

Kelly Kay

Background: In 2018, the Ministry of Health and Long Term Care (MOHLTC) asked the Provincial Geriatrics Leadership Office (PGLO) to identify and map specialty health care serving older people living with complex health concerns (i.e. frailty) across Ontario. The PGLO agreed on the condition that information collected would be vetted, shared and available to any contributors.

\section{Session Objectives:}

To report on a field-led provincial capacity planning exercise.

To describe the process of engagement with contributors to enable health system planning in concert with front line clinicians.

To share early results from this project and discuss the implications for specialized geriatric services in Ontario.

Methods: A provincial working group defined data elements and service types and created a custom database and data entry portal. A request for 2017/18 program data was distributed to all known specialized geriatric services (SGS) programs in Ontario. In March, maps were made available and webinars were held to validate data and receive feedback from contributors on data limitations and possible uses of the data.

Results: Approximately 150 contributors submitted data for over 500 programs and services reflecting more than 180,000 unique patients, 500,000 patient visits, 2500 clinicians, 200 facilities, and over 60 different service types. Nearly 50 success stories and narrative descriptions of models of care were collected.

Conclusions: The resultant supply, utilization and narrative data provides information about the nature, organization and delivery of specialized geriatric services across Ontario, contributing to gap identification, improved definitions and future health service design for older adults living with frailty.

\section{Advocacy \\ Valerie Scarfone}

Background: This section will describe the role of such integration in the structuring of governance models that can enable clinicians to organize and advocate for frail older adults. The symposium will draw from the experiences of different experts doing such work in integration to stimulate 
dialogue and advocacy towards more integrated care. Advancing the vision and passion of dedicated geriatric specialist to expand organizational mandates to include experts from all specialties to better serve seniors with frailty across Ontario. The newly integrated provincial network has created a new governance framework through grass roots engagement that will ultimately translate to better health care for seniors across Ontario and will create a network that will be positioned to lead the development of new models of care for frail seniors.

Methods: Our presentation will describe the process of evolving a long standing provincial structure into a new governance framework that will position the organization to represent the various stakeholders that support seniors with frailty in Ontario. The presentation will include an overview of the current state and the lessons learned throughout the process that led to the development of a more inclusive, comprehensive provincial structure.

Results: Conference participants will learn how a governance framework contributes to developing a more comprehensive, inclusive provincial structure. Additionally the session will highlight the importance of governance as a foundation for service development, standards and ultimately quality care.

Conclusions: This session will focus on the journey of governance evolution and the importance of stakeholder engagement and co design.

Forging Interprofessional Collaboration and Improving Access to Geriatric Expertise with eConsult: GeriMedRisk

Joanne Ho, Sophiya Benjamin, Lindsay Cox, Jack Bodkin

Background: Older adults are at increased risk of harm from adverse drug events (ADEs), one of the leading causes of death and disability in North America. GeriMedRisk is an interprofessional telemedicine consultation and education service supporting clinicians to optimize medications for their older adults. Leveraging eConsult and telephone, GeriMedRisk provides integrated clinical support and consult-based education from geriatric psychiatry, geriatric medicine, clinical pharmacology and pharmacy within days. We hypothesized that GeriMedRisk was a feasible intervention and its potential to improve prescribing, decrease falls and drug-related hospital visits could be evaluated with a stepped-wedge cluster randomized controlled trial design.

Methods: We conducted a cluster stepped wedge randomized feasibility trial of GeriMedRisk among four LTCHs in the Waterloo-Wellington region from May 1 to December 31, 2017. The primary outcome was the feasibility and acceptability of GeriMedRisk and the study design. ISRCTN clinical trials registry, ISRCTN17219647
Results: During the study period of a total of 4 LTCHs, there were 261 person-years of observation. For LTCH residents with a GeriMedRisk consultation, the mean total number of prescriptions was $17.96+/-5.65$ (Standard Deviation (SD)) pre- and $17.78+/-5.49$ post-GeriMedRisk consult. An average of $0.96+/-5.39$ SD medications per patient were decreased or discontinued. The intervention was associated with a decrease in prescriptions, average daily pill count, Beer's Criteria score and number of STOPP medications. Clinician feedback was limited however, highlights included confirmation that the intervention resulted in hospitalization prevention and the consult-based education was valuable.

Conclusions: GeriMedRisk is a feasible interprofessional geriatric clinical pharmacology and geriatric psychiatry telemedicine consultation service and has the potential to decrease polypharmacy and potentially inappropriate prescribing. A larger efficacy study to assess its effects on hospitalizations, falls and prescribing of potentially inappropriate medications is warranted.

\section{Electroconvulsive Therapy in Canada: Emerging Trends in Geriatric Practice: Part 2}

\section{Kiran Rabheru, Peter Chan, Caroline Gosselin}

Background: A relative lack of uniformity in clinical practice and training standards currently exists in Canadian ECT practice and education. Resources to support staffing, space allocation and budgets for Canadian ECT programs are challenged in a competitive health environment.

Method: This second of a two-part workshop will introduce the Canadian ECT Standards (CANECTS) and describe its current application in accreditation for ECT Programs across Canada, including the results of a pilot audits research study, and the recent Ottawa Hospital audit experience. An update on the imminent enhancement of ECT education in Canada via the RCPSC Competency by Design will be discussed. A report from the CPA symposium regarding a potential creation of a national ECT registry will also be provided.

Results: Enhanced knowledge of ECT Standards and the new audit process of ECT programs across Canada will reflect better quality of care in ECT practice, and improved patient safety. Enhancement of ECT education as well as common databases in the form of an ECT registry will lead to better data gathering, improved decision making and resource allocation. Active audience participation leading to the sharing of ECT experience and knowledge will be strongly encouraged in both workshops.

Conclusions: The two workshops will lead to a higher level of skills, knowledge and confidence in the clinical practice, education, research, program evaluation, developing policy in the field of ECT. 


\section{Baycrest Quick Response Caregiver Tool}

Robert Madan, Kenneth Schwartz

Background: Caregivers to people with dementia benefit from tools to help manage responsive behaviours. A number of models (e.g. PIECES, DICE) exist to help caregivers and health care professionals better understand the responsive behaviours and assist with formulation of an intervention. Caregivers require training in effective techniques that can be used to help reduce these behaviours and improve caregiver confidence. The "Baycrest Quick Response Caregiver Tool" was designed to assist formal and informal caregivers in managing responsive behaviours.

Method: The workshop will review the evidence of existing pharmacological and non-pharmacological tools, and the evidence regarding using medications for responsive behaviours in dementia. The "Baycrest Quick Response Caregiver Tool" was designed to be used by caregivers right at the moment of the occurrence of responsive behaviours. Participants will learn how to use the tool using video vignettes.

Results: The "Baycrest Quick Response Caregiver Tool" is being evaluated in family caregivers and is being developed as an e-tool. The current study is examining the impact of the tool on family caregivers. Participants are receiving online training on how to use the tool and impact will be assessed using caregiver burden, mood, and neuropsychiatric scales in a pre/post design as well as focus group data. Preliminary data from this study will be presented.

Conclusions: The "Baycrest Quick Response Caregiver Tool" provides caregivers to persons with dementia with a new tool that assists with responsive behaviours in the moment. Preliminary data in various contexts have demonstrated promising results.

\section{Geriatric Interest Groups: Supporting Trainees with Interest in Geriatrics to Improve the Care of Canadian Seniors}

\section{Amanda Canfield, Ari Cuperfain}

Background: Geriatric interest groups (GIG) are traineeled, faculty-supported groups that encourage trainee interest in older adult care by offering opportunities in education, advocacy, and mentorship. In Canada, 14 medical schools have student-run local GIGs. The National Geriatric Interest Group (NGIG), created in 2010, acts as a centralized body that supports these local GIGs and further promotes interest among medical students through geriatric education and resources. ${ }^{(1)}$ In 2016 the Resident Geriatric Interest Group (RGIG) was created with similar goals but aimed at resident physicians. Various components that are offered by interest groups, such as clinical exposure and access to role models, have been shown to foster interest among medical trainees. $(2,3)$ Indeed, the effect of individual initiatives facilitated by GIGs in promoting interest among medical trainees have been studied.(4) In 2018, members of the NGIG and RGIG conducted a survey that was distributed to current and past members of local GIGs and NGIG to determine the impact these groups had on shaping the career trajectory of their members. ${ }^{(5)}$ The majority of respondents indicated they would likely have a geriatrics-focused medical practice and further $45 \%$ of respondents indicated GIG/NGIG afforded strong mentorship. ${ }^{(5)}$ This interactive workshop will allow trainees to learn more about GIGs including a discussion around the impact they may have on the career trajectories as well as an overview of the opportunities available to trainees through GIGs. It will also include opportunities to provide input to executive members of NGIG/RGIG regarding potential future directions related to resource development for trainees interested in geriatric psychiatry.

References:

1. Guo S. History of the National Geriatrics Interest Group. The National Geriatrics Interest Group Publication. 2017;5:14-17.

2. Drickamer MA, Levy B, Irwin KS, Rohrbaugh RM. Perceived needs for geriatric education by medical students, internal medicine residents and faculty. Journal of general internal medicine. 2006;21:1230-1234.

3. Torrible SJ, Diachun LL, Rolfson DB, Dumbrell AC, Hogan DB. Improving recruitment into geriatric medicine in Canada: findings and recommendations from the geriatric recruitment issues study. Journal of the American Geriatrics Society. 2006;54:1453-1462.

4. Haque AF, Soong DG, Wong CL. Assessing the impact of a Geriatric Clinical Skills Day on medical students' attitudes toward geriatrics. Canadian Geriatrics Journal. 2014;17:12.

5. Perrella A, Cuperfain AB, Canfield AB, Woo T, Wong CL. Do Interest Groups Cultivate Interest? Evaluating Career Trajectories Among Geriatric Interest Group Participants. (manuscript in progress).

Technology Supporting Resilience in Dementia: PatientFacing and Provider-Facing Innovations.

Andrea Iaboni, Arlene Astell, Shital Desai

Background: Technology use is increasing in dementia care including providing recreation, exercise, social engagement, cognitive remediation, and assisting in daily life for individuals with dementia. Care providers use technology for communication, assessment, monitoring, and safety. In all cases, research and evaluation of the technology is essential to ensuring that the intervention preserves the personhood of people with dementia.

Method: In this interactive workshop, Dr. Astell will demonstrate her research into applications of mainstream 
technologies (tablets, game systems) plus development of novel applications to empower people living with dementia, their families and care providers. Dr. Iaboni will discuss the development of environmental, passive health status and behaviour monitoring technologies, and how they might be applied to improve clinical care and decision-making in residential care settings. Dr Desai will present research underway into capabilities and potential of emerging technologies, particularly augmented and virtual reality (mixed-reality) systems.

Results: Patient-facing technologies have been shown to be effective for delivering engaging pastimes, maintaining relationships and improving cognition and quality of life. Provider-facing technologies are acceptable to providers, patients, and families, although more work needs to be done to improve the accuracy of models and the feasibility of the technology. Participants will have the opportunity to trial some of the technologies discussed.

Conclusions: Technology can be used to promote resilience and well-being in people with dementia and their families as well as providing opportunities for its development to promote safe and quality care, and to support the resilience of healthcare providers.

\section{Agitation in Dementia: Advances in Measurement Based Care}

Zahinoor Ismail, Adelaide De Mauleon, David Miller, Paul Rosenberg, Maria Soto, Constantine Lyketsos

Background: Research and clinical work in agitation has been hampered by a lack of agreed upon definition for agitation. Clinical response has been measured as a function of overall clinical impression, or improvement on either agitation specific rating scales or agitation domains of general psychopathological measures. In 2015, the International Psychogeriatric Association developed a definition for agitation to help facilitate research in the field. Important features of the definition are the requirement of distress due to the behaviours, and the breakdown of agitation into three domains: excessive motor activity, verbal aggression, and physical aggression. However, despite the development of the criteria, there are no definition specific measurements.

Method: We describe the modified Delphi process for applying the IPA agitation definition to the Cohen Mansfield Agitation Inventory, and the Neuropsychiatric Inventory clinician rating scale.

Results: We present the IPA definition modifications of the CMAI and NPI-C, and initial impressions from clinical use of these scales.
Conclusions: IPA agitation domain-specific measures are an important advance in measurement and management of agitation in dementia. Next steps include validation of the modified scales in clinical and research cohorts, and assessing the differential responses of the overall agitation measure and the individual domains. Domain specific interventions are an important advance in agitation management, and these CMAI and NPI-C modifications may help this advancement.

\section{Behavioral Support Teams in Acute Care: Lessons Learned in 5 Years with Potential for Scaling Up}

Kiran Rabheru, Vera Hula, Nadine Sebahana, Margaret MacKenzie-Neil

Background: People with dementia are frequently admitted to acute general hospitals a challenging environment for them. Up to $75 \%$ of people with dementia admitted to the acute hospital will experience BSPD with or without delirium. The Ottawa Hospital (TOH) is the first major acute care hospital funded by the Ontario Behavioral Support (BSO) Program with over 5 years of experience. Based on this experience, the Champlain LHIN has scaled the program up to include several other regional acute care hospitals. This session will present data from the $\mathrm{TOH}$ experience including preliminary results from a QA survey of all internal and external stake holders as well as patients and families. Factors that would allow such a program to successfully be scaled up will be discussed.

Method: Clinical and administrative metrics from the $\mathrm{TOH}$ database will be presented highlighting demand, utilization, effectiveness, efficiency, and safety. We will present preliminary data from systematic quality assurance projects to solicit valid and anonymous feedback from internal and external stakeholders as well as patient and family experience will be presented. Factors that may lead to successful scaling up of such an initiative will be explored.

Results: This presentation will be focused on systematically collected data and information over 5 years of TOH's BSO team in acute care. We will present successes, failures, and opportunities encountered during this journey. A systematic QA project results of patient / family experience as well as 360 stakeholder feedback will be used to discuss future planning for scaling up.

Conclusions: BSO teams in acute care settings are a vital tool to provide optimal care to complex older patients who have multiple medical / surgical as well as mental health needs. The teams also provide opportunities for capacity building, training, and improving care across the spectrum of acute care settings. The future expansion of such teams need careful planning and judicious allocation of resources. This presentation will highlight some lessons learned to consider future planning. 
Exercise Intervention for Late-Life Depression: A Systematic Review and Meta-Analysis of Randomized Controlled Trials

\section{Sivan Klil-Drori, Adi Klil-Drori, Shamira Pira, Soham Rej}

Background: There is increasing prevalence of late-life depression world-wide. Due to many benefits of non-pharmacological treatment in aging, and the contribution of exercise to brain health, mental health and general health, it may be a promising intervention for late-life depression. We aimed to estimate the association of exercise intervention with a reduction in depressive symptoms in older adults.

Method: We searched MEDLINE, MEDLINE RCTs, PsycINFO, and EMBASE through December 2018 for randomized clinical trials comparing exercise with a nonactive control condition in adults aged 60 or older. Major inclusion criteria were a supervised, moderate-to-vigorous exercise meeting American College of Sports Medicine recommendations for older adults, and the assessment of depressive symptoms as primary outcome. Random-effects models were used to pool standardized mean differences (Hedges $g$ ) in depressive symptoms across studies.

Results: Eight studies involving 1,187 participants were included; mean participant age was 82.7 years. Moderate-tovigorous exercise intervention was associated with a medium effect size of 0.63 ( $95 \%$ confidence interval $=0.21$ to 1.05 ; $\mathrm{z}=2.95 ; \mathrm{P}=0.003$ ) in reducing depressive symptoms. However, there was considerable heterogeneity $(\mathrm{T} 2=0.25, \mathrm{Q}=31.04$, $\mathrm{P}<0.0001 ; \mathrm{I} 2=77.4 \%$ ) in observed effect across included studies. Age appeared to contribute to heterogeneity, while cognitive function did not. The reporting of adherence and fitness metrics was inconsistent, and the overall risk of bias was deemed high for all studies.

Conclusions: A moderate reduction in depressive symptoms was seen with exercise among older adults. Nevertheless, exercise interventions for late-life depression should likely be tailored to specific populations among older adults.

\section{Problem Solving Therapy Workshop}

Dallas Seitz, Patricia Areán

Background: Depression is common among older adults and psychotherapy is frequently recommended as a treatment for depression in this population. Problem Solving Therapy (PST) is a short-term, evidence-based treatment for depression which is being increasingly studied in older adults with depression and other mental health conditions. PST is one of the most well supported psychotherapies for older adults with depression and the techniques used in
PST are generalizable to the management of other mental health conditions and management of stressful life events. This course will provide attendees with information on the background and history of Problem Solving Therapy along with common strategies employed in PST. Didactic lectures coupled with interactive facilitated role-playing will be used to illustrate the key therapeutic strategies employed in PST and provide attendees with hands on experience with PST that can be applied in clinical practice. Adaptations of PST for different health systems and clinical programs will be reviewed along with recent adaptations of PST. This workshop will be led by Dr. Patricia Arean (University of Washington) who is the worlds expert in Problem Solving Therapy for older adults with depression and Dr. Dallas Seitz (Queen's University) a geriatric psychiatrist with experience in using PST in Canada.

\section{A Review of 2018/2019 Top Papers in Geriatric Psychiatry}

Andrea Iaboni, Zahinoor Ismail, Damien Gallagher, Dallas Seitz

Background: In 2018, the Canadian Academy of Geriatric Psychiatry responded to member needs assessment by developing a "top papers" session, reviewing and appraising recent literature of interest.

Method: Four geriatric psychiatrists will each select two papers published between July 2018 and June 2019. Paper selection will be based on papers of interest that have changed the presenter's clinical practice or approach to research.

Results: Each of the papers will be synthesized by the presenters, and a critical appraisal of each paper will ensue.

Conclusions: The presenters will conclude each paper description by discussing the impact on clinical care or research, and a group discussion will encourage attendees to reflect on the content of the papers, the state of the literature in geriatric psychiatry, and on critical appraisal.

Creating Safer and More Welcoming Continuing Care Services for Sexual and Gender Diverse People (LGBTQ2S+)

Sophie Sapergia

Alberta media and patient feedback reports that continuing care LGBTQ2S + clients feel the need to hide their true identify to avoid bullying and harassment and to feel safer while in care; to receive equal care services. Alberta Health Services collaborated to develop resources to support healthcare providers to create safer and more welcoming continuing care services. Sexually and gender diverse clients will feel safer and more welcome to live their lives authentically. 
Awareness Resources to Support Healthcare Providers to Create Safer and More Welcoming Continuing Care for Sexual and Gender Diverse (LGBTQ2S+) Clients

\section{Sophie Sapergia}

Background: Many older LGBTQ2S+ clients have experienced poor treatment in their lifetimes. Many grew up in a time when identifying as LGBTQ2S+ was considered a criminal offence or a mental health condition. Many suffered social isolation, family rejection, abuse, job loss or even imprisonment. Some LGBTQ2S+ clients have experienced stigma and discrimination when trying to access healthcare services. In Canada, $13 \%$ of Canadians identify as sexual and gender diverse, including over 500,000 Albertans and 15,000 seniors. Alberta media and patient feedback reports that some continuing care clients who identify as LGBTQ2S+ feel the need to hide their true identify to avoid bullying and harassment and to feel safer while in care, and to receive equal care services. The overall goal of this Alberta Health Services initiative to develop awareness resources for continuing care healthcare providers was to increase awareness and knowledge for creating safer and welcoming spaces for sexual and gender minority (LGBTQ2S+) people in Alberta continuing care settings, including home care, designated supportive living, and long-term care.

Methods: Many older LGBTQ2S+ clients have experienced poor treatment in their lifetimes. Many grew up in a time when identifying as LGBTQ2S+ was considered a criminal offence or a mental health condition. Many suffered social isolation, family rejection, abuse, job loss or even imprisonment. Some LGBTQ2S+ clients have experienced stigma and discrimination when trying to access healthcare services. In Canada, 13\% of Canadians identify as sexual and gender diverse, including over 500,000 Albertans and 15,000 seniors. Alberta media and patient feedback reports that some continuing care clients who identify as LGBTQ2S+ feel the need to hide their true identify to avoid bullying and harassment and to feel safer while in care, and to receive equal care services. The overall goal of this Alberta Health Services initiative to develop awareness resources for continuing care healthcare providers was to increase awareness and knowledge for creating safer and welcoming spaces for sexual and gender minority (LGBTQ2S+) people in Alberta continuing care settings, including home care, designated supportive living, and long-term care.

Results: Education participants reported $85 \%$ better awareness and $84 \%$ improved knowledge to create safer and more welcoming care environments. Project volunteers reported the project materials would make a meaningful difference to the lives of sexual and gender diverse patients. Recommendations were made for additional resources to support continuing care provider organizations to implement the awareness resources, adding more scenario-based examples, as well as developing resources for clients and their families. These additional materials are currently under development.

Conclusions: In Alberta, resources have been developed to start the conversation and support healthcare providers to create safer and more welcoming continuing care services for clients who identify as sexual and gender diverse. Initial feedback through participant surveys has been generally positive.

Supporting Safe, Healthy, and Dignified Expressions of Sexuality in Continuing Care: The State of the Science and the Alberta Experience

Julia Brassolotto, Lisa Howard

Background: We have explored how residents' sexual expression is supported in Alberta's continuing care homes. In the presence or absence of policy, managers and those whom they consult (clinical ethicists, best practice/geriatric assessment teams, social workers, and others) have the authority to determine site level responses to sexual expression and shape the culture of a care home.

Methods: We received ethics and operational approvals for this research. We used a qualitative exploratory design to identify how sexual expression is navigated in continuing care settings. We conducted semi-structured interviews with 28 participants over the telephone or in person.

Results: Our findings confirmed and extended the themes from the existing literature and highlighted experiences specific to the Alberta context. Key themes include: (1) broad definitions of sexual expression and its place in continuing care homes, (2) structural features that influence how leaders navigate residents' sexual expression, (3) interpersonal dynamics, and (4) exemplary cases of support.

Conclusions: Our research provides insight into the experiences of continuing care leaders with navigating resident sexual expression. Participants were unequivocal that diverse sexual expressions enrich residents' quality of life. Our findings are optimistic and fraught. There is urgent work to be done. While there is tremendous interest to ensure residents' sexual expression is safe and healthy and there are systemlevel assets for consultation and education, it is clear that additional resources are needed for supporting a comprehensive, person-centered approach.

Creating a Continuing Care Environment Where Sexual and Gender Diverse Clients Feel Safer and More Welcome to Live Authentically

Krista Chapman 


\section{CAGP-CCSMH ABSTRACTS}

Background: Carewest, Calgary's largest public provider of residential continuing care and rehabilitative services, began the journey to look at their services and processes to support sexually and gender diverse clients to feel safer and more welcome to live their lives as they identify. Some clients were comfortable to share their diversity, others took their time and it is unclear if there are other clients that were hiding their sexual and gender diversity. Carewest made it a priority to enhance services and undertook a quality improvement initiative.

Methods: Carewest incorporated the learnings from the four AHS continuing care tip sheets and other developed resources to enhance their continuing care service delivery. Areas of focus included leadership engagement, staff education and environmental modifications.

Results: A soft roll-out of the concepts and processes started with leadership role modeling and staff education. The rate of adoption of the concepts and strategies were unique for each leader, healthcare provider and resident.

Conclusions: Residents, families, visitors, healthcare providers and leadership at Carewest have increased awareness, understanding, knowledge, and confidence to create safer and more welcoming care for sexually and gender diverse clients.

\section{Capacity Assessment in Older People: Interactive Case Workshop}

Karen Reimers

Background: As the population ages, clinicians are increasingly faced with questions about the mental capacity of older individuals, including capacity to consent to or refuse treatment, drive, manage independently at home, and more. These scenarios are commonly seen across outpatient clinics, hospitals, nursing homes and other settings. The consequences of determining a patient's mental capacity can be far reaching. Older patients and their families can benefit from a practicing clinician's competent assessment of mental capacity.

Method: Updated with new material in 2019, this interactive case-based workshop will engage participants in a lively discussion of common clinical scenarios involving assessment of mental capacity in older adults. Each case is based on clinical experience with older adults in a general psychiatric practice. Cases are selected to reflect common scenarios that clinicians are likely facing in their daily practice, whether they work in a primary care or psychiatric specialist setting.

Results: We will identify the most common areas of clinical difficulty for practicing clinicians. The range of cases will include assessment of capacity to make medical decisions, manage finances, driving capacity, the role of psychiatric and medical disorders affecting mental functioning, and more.
Conclusions: Following each case discussion, we will review pertinent clinical and ethical dimensions for that case. We will highlight recommendations and practical strategies for clinicians to implement in their practice. References for each case will include peer reviewed literature in this evolving field.

Integrating Applied Behaviour Analysis into a Collaborative Practice Model for the Treatment of Responsive Behaviours in LTC Residents living with Dementia

Nick Feltz, Adele Loncar, Timah Black-Geisterfer

Background: For decades Applied Behaviour Analysis (ABA) has sustained its procedural efficacy and social validity as a non-pharmacological treatment for behavioural symptoms across diverse populations. However, its application within dementia care is notably sparse. The Champlain Behavioural Support System received enhanced funding and was directed to the development of the Geriatric Behaviour Therapy Program at The Royal Ottawa. The Behaviour Therapists (BTs) are now integrated within a collaborative model of service delivery in the Geriatric Psychiatry Outreach and BSO teams in LTCHs across the Champlain LHIN.

Method: Multiple variables correlated with the onset of responsive behaviours were identified through a behavioural and environmental assessment completed by a BT. A collaborative treatment package consisting of an environmental change aimed to reduce behavioural precursors, evidencebased staff training, and polypharmacy monitoring was utilized to treat the correlated environmental variables contributing to responsive behaviours therefore preventing an in-patient admission for a resident in LTC.

Results: Following the collaborative treatment package there were evident decreases in responsive behaviour thus successfully maintaining the residents placement in her current LTCH.

Conclusions: This discipline within Geriatric Psychiatry embodies the principles of ABA and maintains a collaborative approach while focusing on building capacity within LTC to address socially significant behaviour, and improve the quality of life for residents living with dementia. New to dementia care, this discipline is yielding promising results. An exhaustive ongoing evaluation of this new program is underway.

\section{A Structured Teleconsultation and Coaching Project as a Way to Improve Care for Behavioral and Psychological Symptoms of Dementia (BPSD) in Quebec}

Marie-Andrée Bruneau, Anne Bourbonnais, Nathalie Bier, Caroline Ménard, Laurence Villeneuve, Stéphanie Daneau, Catherine Dubé 
Background: Behavioral and psychological symptoms of dementia (BPSD) are highly prevalent and have considerable impacts. There is still insufficient knowledge, suboptimal management and excessive use of psychotropic medications in many clinical settings. Given the low availability of specialized staff and the remoteness of some healthcare settings, telehealth might be an efficient way to disseminate knowledge and competency in BPSD care.

Method: Geriatric psychiatry specialists at the Institut Universitaire de Gériatrie de Montréal (IUGM) offered a structured training program to distant community care teams through teleconsultation. It involved setting up a BPSD team in each participating community. The program proposed a 2-day formal training followed by a monthly complex BPSD case teleconsultation. Since 2011, 9 agreements have been signed between the IUGM and regional partners. One of these partnerships, with Magdalen Islands health and social services centre (IDLM), included a formal research project. The objectives of this project were to document the effects of the service as well as the implantation process.

Results: Fifty-seven teleconsultations were done over 6 years. Patients were on average 80 y.o., ( $75 \%$ women), mostly living in long term care (72\%). Results from IDLM project showed clinical benefits ( $\mathrm{N}=12$ : decrease of $35 \%$ NPI-R, improvement on CGI), as well as high satisfaction level. Eleven open interviews were completed. Professionals felt that the project helped to define a better care trajectory for BPSD, improved their sense of competency and give them relief from helplessness and isolation.

Conclusions: These results suggest that teleconsultation is a good way to disseminate expertise concerning BPSD.

\section{Aging with Resilience-Surviving, Responding and Adapting}

Mary Hynes, Christopher A. Klinger, Raza M. Mirza

Background: The increasing population of older adults is creating social, financial and health systems pressures that may be better addressed through increased resilience and multi-modal interventions. The evidence highlights that reducing the incidence of cognitive decline and diseases of aging using unimodal interventions have shown minimal effects. A scoping review of multimodal interventions was conducted to better understand why interventions using two or more interventions may be better suited for older adults. The two most promising multimodal interventions, employing five modalities each, were the Finnish Geriatric Intervention Study to Prevent Cognitive Impairment and Disability (FINGER) and the Fountain of Health Initiative for Optimal Aging (FoH). FINGER was a 2-year clinical trial that is being continued and extended. FoH engaged clinicians in working with patients to change behavior.
Method: Building on the hypothesis that older adults who participate in multimodal interventions and assisted goal setting may be more resilient, the aim of this study is to identify potential methods for creating multimodal interventions. This proof-of-concept project is recruiting older adults from two community health centres to attend an information session and be guided into choosing one SMART (specific, measurable, attainable, realistic, time-based) goal and to begin implementing multimodal interventions within a one-month period.

Results: Systemic content analysis will be used to compare participants' goal behaviours, previous adaptive actions and demographic factors to show increased resiliency through guided goal setting.

Conclusions: This proof-of-concept aims to demonstrate that non-specialist community workers can engage older adults in using SMART goal setting for improved self-resilience/ well-being.

\section{Psychiatric, Motor and Autonomic Effects of ECT in Depressed Parkinson's Disease Patients}

Nicholas J. Ainsworth, Isaac Rodin, Je Hun Sung, Fidel Vila-Rodriguez

Background: Parkinson's disease (PD) is a neurodegenerative condition comprising a significant source of morbidity in older adults. In addition to the motor symptoms of the disease, so-called non-motor syndromes, including depression, are increasingly recognized to contribute to the burden of disease. While some evidence exists for antidepressants as treatment, many patients require alternatives due to lack of efficacy or tolerability concerns. Convulsive neurostimulation treatments including electroconvulsive therapy (ECT) are a promising avenue of treatment for many patients; however, high-quality evidence for ECT remains limited in this population.

Objective: Conduct a retrospective study on the effects of ECT in depressed PD patients in a local setting, to provide preliminary data for a future prospective trial.

Method: Clinical data were retrieved from medical records at St. Paul's Hospital in Vancouver, BC, pertaining to depressed PD patients receiving ECT between 2016 and mid-2019. Demographic, medication, ECT treatment, and clinical outcome data, including rating scales if available, were recorded. A descriptive analysis was planned for the purposes of hypothesis generation.

Results: A total of eight patients met inclusion criteria and were analyzed. A majority (88\%) had significant improvement in depressive symptoms following ECT, and of these $71 \%$ went on to receive maintenance treatment. Although motor rating scale data were infrequently recorded, there was a notable decrease in median levodopa dose in patients 


\section{CAGP-CCSMH ABSTRACTS}

who completed a full course of ECT (-250mg), suggesting possible improvement in motor function. Discontinuation of ECT due to tolerability concerns occurred in $25 \%$ of patients. Analysis of autonomic response during ECT showed more within-treatment volatility in both blood pressure and heart rate among patients who discontinued, which did not appear to be confounded by their shorter ECT courses.

Conclusions: This study demonstrates the feasibility and plausible efficacy of ECT as a treatment for depression in PD patients, and provides plausibility to the hypothesis that it may improve motor function. Our autonomic data suggest a possible cause of tolerability concerns, which may be predicted by a more volatile autonomic response to ECT. A follow-up prospective trial is planned.

\section{Ageism Perceptions and Transitions}

\section{Shabbir Amanullah, Karen Cassells, Sean Welbourn, Amy Swayze, Mark Dager, Michelle Worsfold}

Background: People of all ages hold attitudes related to aging; every single one of us faces the challenge of growing older on a moment-by-moment, daily basis (Elder Abuse Awareness 2019) and although many will demonstrate similar patterns of advancing age, biological and chronological measures differ. The psychological concept of resilience is rooted in early developmental patterns and may sometimes be seen as a process rather than a personal characteristic or trait of character (Windle G 2010). Along the continuum of human development there are often strong emotional and social connections attached within relationships whether it be family, friends, neighbors, educators, clergy or co-workers. Milestone attainments associated with "growing up" are not often labelled as "aging or growing old" yet are measured by life events such as; learning to walk, beginning school, voting, joining the workforce and retiring. "Ageism" often attaches a negative connotation; elderly individuals may on occasion be viewed as helpless, weak and no longer able to meaningfully contribute to society. Resilience in health care delivery for the elderly client manifests when facing an adverse health event; elderly hospitalized clients may experience isolation and vulnerability due to their own aging process, encountering difficulty regaining baseline function. Interaction with old persons and personal outlooks toward growing old may well be influenced by one's general aging-related beliefs. At times with "better health care" the old are forced to give up their autonomy and take on the passive role of a patient connecting the notion of aging as a frail condition (Ferrucci, 2006).

Method: Resilience by definition is: an ability to maintain healthy psychological functioning when faced with a tragedy, natural disaster, health concern, relationship, work, or school problem, resilience is how well a person can adapt to the events in their life. A person with good resilience has the ability to bounce back more quickly and with less stress than someone whose resilience is less developed (Cohen 2018). Successful aging and resilient aging examine how an individual process and adapts significant life events and difficult experiences regardless of socio-economic background, personal experience or social environments (MacLeod 2016). In order to examine perceptions in this age group a need exists for development of a trusting rapport whether it be with family, friends or health care providers and at times law enforcement . Older adults are often reluctant to discuss the nature or extent of a problem as such a disclosure may affect their autonomy. Older adults for the most part tend to minimize the gravity of situations especially in the presence of abusive situations. Community services to manage and support elderly clients experiencing abuse/alleged abuse may not be readily accessible or able to support elderly clients based on inaccuracy of the actual number of older adults reporting the concern. Elderly clients may also not know how to access available resources . Personhood enhancement through effective community partner relationships supporting older adults bring collaborative efforts forward to support the individual client in advance of the "crisis" based need for support.

Results: The "silent generation" (those born between 19251945) traditionalists by virtue of the era they were born; learned a practical optimism as influence from their youth do not always come forward when facing challenging situations such as declining and changing health, debility and potential abuse. Abuse in itself should not come with ageing but often it does. The goals of reducing stigma in this age group poses a significant set of challenges. The fear of disclosing any form of actual or potential abuse may often impact the ability for an elderly client to return home, transition to retirement home or to long term care from hospital where most often elderly clients present at the immediate time of crisis. Length of stay becomes influenced when the elderly client is hospitalized for any alleged or actual abuse and readmission to hospital may occur upon transition to another environment. Safety concerns weigh heavily on system delivery of care. A successful placement and discharge transition occurred for an elderly client in the region with partnered support offered through a transition model generated in the LTCH sector within the county.

Conclusions: A lot can be learned from old people and the normal ageing process. Old age does not need to be a time of ill-health, loneliness, abuse or neglect. The perception of when "old age" begins is a unique and individual experience and ones own age related beliefs.

The Use of Complementary and Alternative Medicine for Pain Relief in the Elderly: An Investigational Analysis of Seniors Residing in an Independent/Assisted Living Facility

Carol Cameletti, Carly Buckner 
Background: Physically, seniors with chronic pain experience symptoms ranging from discomfort with a limited range of motion to intolerable pain and impairment. Psychologically, seniors experience frustration and confusion regarding a remedial process, and hopelessness if the pain is incessant. Studies indicate that CAM use is highly prevalent among older adults, health care-prescribed failure to control pain is a primary motivator for CAM use and that elders are typically satisfied with the benefits from CAM treatment. With the potential to alleviate physical pain, patients with chronic pain have adequate reasons to search beyond their physicians' solutions.

Method: Participants were interviewed by research assistants who helped complete two questionnaires: 1) a self-assessment on pain (Brief Pain Inventory, BPI) (Appendix A), and 2) CAM use for pain (Appendix B).

Results: Results from the CAM-use questionnaire showed that participants did try alternative therapies to help manage their pain. Participants were asked to list all treatments and/ or medications that they had been using for pain management. There was a significant range of treatments listed by the participants, such as: nothing, Tylenol, St-Johns' Wort, Glucosamine msn, ibuprofen, Lyrica, Aspirin, Fentanyl patch, vitamin E, Quinine sulfate, oyster stew and Diclofenac.

Conclusions: Our findings demonstrate that chronic pain could be a substantial problem for community-older adults living in Sudbury. Presently there are no clinical practice guidelines for alternative treatments for the significant relationships between chronic pain and various measures of physical health, and clinicians do not consistently assess for chronic pain using validated and reliable instruments.

Educational Opportunities of Interest to Psychiatry Resi-
dents Considering Subspecialization in Geriatric Psychiatry Amanda Canfield, Karen Saperson

Background: There is currently an identified shortage of geriatric psychiatrists within Canada. While studies have examined factors that contribute to the development of interest in geriatric psychiatry amongst psychiatry residents, to our knowledge no studies have examined factors that may further nurture this interest once it is established. The first goal will be to explore what psychiatry residents considering subspecialization in geriatric psychiatry would find helpful to decision-making on where to pursue training including what they are looking for in programs. The second goal will be to identify educational opportunities and resources that residents considering geriatric psychiatry feel would encourage their interest.

Method: The first goal will include focus group(s) held with psychiatry residents within the Department of Psychiatry and Behavioural Neurosciences at McMaster University that have expressed interest in geriatric psychiatry. Consideration will also be given to reaching out to psychiatry residents at other universities within Canada if further input is necessary. The second goal will include discussion at the CAGP-CCSMH 2019 Annual Scientific Meeting during a workshop entitled "Geriatric Interest Groups: Supporting trainees with interest in geriatrics to improve the care of Canadian seniors". The use of a survey disseminated to Canadian psychiatry residents will be considered if further input is sought.

Results: Results will contribute to information about what residents with interest in geriatric psychiatry are seeking in educational opportunities. This information can be used to direct the activities of geriatric interest groups and the CAGP in their future efforts.

Conclusions: To be added upon completion of project.

\section{The Challenge of Sleep Disorders in Persons with Demen- tia: A Review of Current and Proposed Treatments}

Antonia Cappella, Glen Baker, Lawrence Pawluk

Background: Age-related sleep changes including advanced sleep phase, increased sleep latency, decreased total sleep time, increased sleep fragmentation, decreased slow-wave sleep, reduced REM sleep and reduced sleep efficiency are common amongst older adults. Sleep disturbances are even more prevalent in persons with dementia (PWD). It is estimated that $>40 \%$ of PWD experience sleep disturbances including insomnia, daytime somnolence, circadian rhythm disturbance, REM sleep behaviour disorder and sleep disordered breathing. Over the past 15 years there has been growing research in the field of sleep disorders in PWD.

Method: MEDLINE (including PubMed), Embase and CINAHL Plus searches were performed for English language publications up to January 2019 with no limit set on date of initial publication. The search generated 4270 articles (excluding repeats). All titles were reviewed for relevance and 214 articles reviewed in their entirety.

Results: There are a number of studied pharmacological interventions for the management of sleep disturbances in PWD; these interventions include use of melatonin, cholinesterase inhibitors, antipsychotics, sedative-hypnotics, stimulants and herbal supplements. In addition, a number of non-pharmacological interventions including bright-light therapy, exercise, cranial stimulation, socialization programs, sleep hygiene and multi-modal approaches thereof have been examined for the management of sleep disorders in PWD.

Conclusions: Despite growing interest in the field of sleep disturbances in PWD there is a relative paucity of evidence for both the pharmacological and non-pharmacological 


\section{CAGP-CCSMH ABSTRACTS}

management of such conditions. Further research, particularly randomized control trials, is required before definitive recommendations can be made regarding the management of sleep disturbances in PWD.

\section{Emerging Suicide Trends in a Tribal Population in South India}

Susmita Chandramouleeshwaran, Mahantu Yalsangi, Bhawna Sharma

\section{Background:}

- Suicidal behavior is a major contributor to morbidity and mortality worldwide

- India ranks 43rd in international suicide incidence list1

- Over $90 \%$ of all suicides are believed to occur in the context of mental disorders2

- Suicide was a rarity within the Adivasi (Tribal) community of Gudalur, South India

- High suicide mortality rate observed since past two decades despite presence of community mental health program

This Study Aims To:

- study incidence and socio-demographic factors of completed suicide in the tribal population

- understand the risk factors and reasons for completed suicides, and to compare with available data

\section{Method:}

- Mixed methodology: Quantitative and qualitative component

- Sample: Catchment population of 17,965 people from four different tribes

- Qualitative data: 10 Semi-structured In- depth interviews with first degree family members of persons who had completed suicides over last one year

\section{Results:}

- 88 completed suicides reported during the study period, suicide incidence rate of $81.6 / 100000$ compared to 10.6/100000 national rate

- Suicide most common cause of mortality in the age group of 15-45 years

- Third most common cause of mortality (after cancer and stroke) in study population

- Most at risk are older people in the population and people from Mullukurumba tribe, who are most integrated with the non indigenous population

- Common modes of committing suicide - hanging and pesticide consumption

- Major causative themes emerging are interpersonal problems, untreated mental illness, and alcohol use

\section{Conclusions:}

- Suicide rate among the tribal population studied much higher than the national average, especially in geriatric population, and represents an emergenvy
- More than half of the suicides as viewed in the qualitative findings, were preventable

- There is an urgent need for community based education on the suicide epidemic, to promote equity in health, and address the alcohol use problem.

\section{Effectiveness of a Dyadic Chair Yoga Intervention for Re- ducing Stress in Dementia Patients and Their Caregivers}

Elena Dikaios, Vasavan Nair, Marouane Nassim, MarieAndrée Bruneau, Soham Rej

Background: Dementia is associated with stress, anxiety, and depression in both patients and caregivers. Yoga may be a viable therapeutic modality to address these challenges, as it has been shown to decrease stress and improve anxiety and depressive symptoms in various populations. While there is preliminary data on dyadic exercise interventions for dementia patients and their caregivers, to date no research has examined the effectiveness of a dyadic yoga intervention.

Method: We propose an 8-week single arm pilot trial of a dyadic chair yoga intervention for patients and their caregivers $(\mathrm{n}=30-40)$. The primary outcome is perceived stress (Perceived Stress Scale (PSS)), while secondary outcomes include symptoms of depression (Patient Health Questionnaire (PHQ9)) and anxiety (Generalized Anxiety Disorder 7-Item Scale (GAD7)). Exploratory outcomes include caregiver burden (Zarit Burden Inventory (ZBI)), quality of interactions (Dyadic Relationship Scale (DRS)), well-being (12-Item WellBeing Questionnaire (W-BQ12)), mindfulness (Five Facet Mindfulness Questionnaire (FFMQ)), and self-compassion (Short Form of the Self-Compassion Scale (SCS-SF)).

Results: The protocol of this study will be presented at CAGP 2019 in Banff. Results will be ready by mid-2020.

Conclusions: We anticipate that this novel dyadic chair yoga intervention will improve stress, as well as depression, anxiety, and other outcomes, in dementia patients and their caregivers. If successful, this study provides crucial pilot data for a larger definitive randomized controlled trial. Future studies can also assess whether this intervention may improve direct and indirect health-care costs.

The Effectiveness and Safety of Electroconvulsive Therapy for Treatment Refractory Agitation or Aggression in Major Neurocognitive Disorder

Claire Docherty, Peter Chan, Robyn E. Waxman, Sarah Elmi, Mafalda Musacchio, Michael Wilkins-Ho, Simon Woo,

Background: Neuropsychiatric symptoms (NPS) of those with Major Neurocognitive Disorder (MNCD) include the 
responsive behaviours of agitation and aggression. ECT has shown some effectiveness based on retrospective studies and one open label prospective study. We hypothesized that ECT will reduce NPS between baseline and after treatment in those with medication refractory behaviours.

Method: This Canadian prospective multi-centre study included MNCD inpatients admitted to geriatric psychiatry units for the management of refractory NPS. All treatmentrefractory participants enrolled suffered from advanced MNCD. We conducted the Neuropsychiatric Inventory (NPI) and the Pittsburgh Agitation Scale (PAS) at baseline, during and up to 12 weeks after the ECT course. A bitemporal or bifrontal ECT series based on dose titration to 1.5-2.5 times seizure threshold was administered.

Results: Data was collected for 26 patients with a mean age of 74.27 most categorized with advanced MNCD using FAST rating scale (stage 6 and 7). The data showed a drop in mean NPI (from 61.31 to 25.69 [ $n=26, p<.000]$ ). Mean NPI agitation subscale dropped from 7.40 to 2.92 . [ $\mathrm{n}=25, \mathrm{p}$ $<.000]$. Mean NPI aggression subscale dropped from 7.45 to $1.10[\mathrm{n}=20, \mathrm{p}<.000]$. Mean PAS dropped from 8.83 to 4.30 $[n=23, p<.001]$. No patient has dropped out due to intolerance of ECT and no serious adverse events or deaths have been attributed to ECT.

Conclusions: In this naturalistic study to date, ECT is a safe and effective treatment for certain NPS in people with MNCD. This can translate into improving quality of life and lessening caregiver burden.

\section{Special Considerations for the Use of Lithium in Older Age Bipolar Disorder}

Jocelyn Fotso Soh

Background: Lithium is the gold standard treatment for bipolar disorder. It is effective in the management of manic, depressive, and maintenance phases of bipolar disorder treatment. Despite this, the implications of lithium use in the older population remain less understood.

Objective: This critical narrative review aims to better understand the impact of lithium in older age bipolar disorder (OABD), including tolerability and efficacy, based on up to date evidence.

Method: Relevant studies of efficacy, effectiveness, and tolerability any time prior to May 2018 were identified using the PubMed keyword search: "lithium older adult bipolar disorder" and references from recent international bipolar disorder guidelines.

Results: One randomized controlled trial was identified, the GERI-BD (Acute Pharmacotherapy in Late-Life Mania) study.
This study found lithium to be effective in late-life mania and hypomania. The remaining literature examining lithium in $\mathrm{OABD}$ was reviewed, with special considerations highlighted.

Conclusions: In summary, there is a small, yet increasing geriatric evidence base that lithium is effective in OABD. Although there can be adverse effects with lithium, it is generally well tolerated, and there are methods to minimize these risks. Further research would strengthen the evidence base for lithium therapy in OABD. In the meantime, lithium remains the gold-standard treatment for OABD.

\section{Closing the Knowledge-to-Action Gap in Health Promo- tion: A Feasibility Study of Fountain of Health Behaviour Activation Tools in Routine Care to Promote Patient Self- Rated Wellbeing and Health Behaviours}

Amy Gough, Kathleen Singh, Beverley Cassidy, Kara Matheson, Keri-Leigh Cassidy

Background: The Fountain of Health $(\mathrm{FoH})$ is a national health promotion initiative that translates the science of brain health and resilience using cognitive behavioural therapy (CBT)-based behaviour change tools.

Method: FoH's CBT-based intervention was used by clinicians in Nova Scotia, Canada during up to three clinical encounters with patients during routine care. Education about the FoH tools was offered to volunteer clinicians who then used the tools with patients over 50 years of age with no dementia. Clinicians reviewed evidence-based domains of brain health (social activity, physical activity, brain challenge, taking care of mental health and positive outlook) and invited health behaviour activation through goal setting and tracking. Primary outcome measures were patient self-reported Health and Resilience Questionnaire (health attitudes), and goal attainment scale (behaviour change). A questionnaire for clinician feedback was also included.

Results: A total 116 patients received the FoH intervention during routine clinical care by 16 clinicians. A significant difference $(p<0.001)$ was found in patient self-reported social connectedness, interest in learning new things, perceptions of aging well, and physical activity following the FoH intervention. Of 176 goals set, 108 (61.4\%) were completely reached or exceeded. 165 of those goals $(93.8 \%)$ resulted in positive health behaviour change.

Conclusions: FoH's brief CBT-based intervention to promote health behaviour change delivered in routine frontline over a few routine clinical encounters significantly improved patients' self-reported health attitudes and key behaviours known to promote brain health. Larger studies across diverse settings are needed to assess generalizability of these findings. 


\section{The Perception of Patients with Dementia About a Social} Robot PARO in a Hospital Setting

Lillian Hung, Cindy Liu, Evan Woldum, Andy Au-Yeung, Annette Berndt, Christine Wallsworth, Neil Horne, Mario Gregorio, Jim Mann

Background: New technology such as social robots opens up new opportunities in hospital settings. PARO, a robotic pet, was designed to provide emotional and social support for older people with dementia. This project aims to explore the perception of persons with dementia about the role of PARO in a hospital setting.

Method: Video-ethnographic methods were applied in this qualitative study. Patient and family partners were involved in the fieldwork of data collection and analysis. We conducted conversational interviews with ten patients with dementia staying in a geriatric unit and video observations while patients were interacting with PARO in various clinical situations.

Results: Thematic analysis yielded three substantive themes: (a) "it's like a buddy", the robot helps persons with dementia to uphold a sense of self in the world, (b) "it's a conversation piece", the baby seal facilitates social connection, and (c) "it's all about love", PARO transforms and humanizes the clinical setting.

Conclusions: Our findings contribute to providing a better understanding of the direct perspectives of patients with dementia on the use of the social robot. Instead of substituting human contact, the social robot complements emotional care and supports our fundamental human need for love.

\section{Behaviour Assessment Tools Currently Used in Long Term Care in Canada: A Survey}

Andrea Iaboni, Marion Penko, Hannah Quirt

Background: Many people living in long-term care (LTC) have behaviour symptoms associated with dementia. At present, there is no standard tool for the measurement of these symptoms in LTC outside of the quarterly resident assessments. Accurate measurement of behaviour symptoms is essential to guide treatment and monitor the effect of interventions. Limitations to existing tools include the lack of training, rater bias and recall errors. These limitations threaten their reliability and validity and create the risk of over- or under-treatment of behaviour symptoms. In this study, we sought to understand which tools are currently used to assess behaviours in LTC and challenges associated with their use.

Method: 300 LTCs across Canada were invited to participate in an on-line survey to establish: which behavioural assessment tools are used most, what supports their use and what makes them challenging.

Results: Of 300 LTCs invited to participate in the survey, 110 responded, with 7 declining to participate in the survey and 103 completing the survey. Nine provinces and one territory were represented by the survey respondents. Homes reported using a mean $2.3+/-1.2$ (range $0-7$ ) tools. The two most commonly used tools are the DOS and CMAI, followed by the ABRA and $A B C$ charting. We were able to identify the most common reasons behaviour assessment tools are used, who completes them and interprets their results, and how these results are used.

Conclusions: There are important opportunities for improving the assessment of behaviour in LTC, including standardization, improved education and the use and development of mobile technology for behaviour assessment.

\section{Healthcare Costs, Healthcare Utilization, and Medical Burden among Older Adults with Schizophrenia}

Waqas Khan, Mulsant BH, Kumar S, Voineskos AN, Rajji TK

Background: In developed countries, the number of older adults with schizophrenia (OAS) aged 55 years and older will soon amount to roughly 1.1 million people by 2025 . Globally, the number of OAS is expected to double between 2014 and 2050 to 10 million people. Addressing the need for greater awareness and management regarding OAS extends beyond geriatric mental health and has considerable public health implications.

\section{Method:}

Objectives: With a scarcity of longitudinal studies examining OAS and few investigating the healthcare and medical burden in a single cohort, over a long duration, and none from a Canadian perspective, our objective is to quantify healthcare utilization, costs, and medical burden in this population compared with healthy age-related controls.

Hypothesis: Older adults with schizophrenia will have greater healthcare utilization, costs, and medical burden when compared with healthy age-related peers.

Study Design: A 10-year longitudinal study.

Setting: Urban academic tertiary mental health center and community living.

Participants: A total of 60 adults, 50 years and older, with schizophrenia and 30 age-related healthy controls.

Measurements: The number of psychiatric hospitalizations, acute psychotic episodes, co-morbidities, and duration of illness 
were taken from psychiatric history records and the Cumulative Illness Rating Scale-Geriatric (CIRS-G). Cognition was assessed using the MATRICS Consensus Cognitive Battery (MCCB), while functional capacity was measured using the USCD Performance-based Skills Assessment (UPSA).

Results: Under preparation, will be ready for the conference.

Conclusions: We will discuss how our results compare to the existing literature and future implications of regarding healthcare and medical burden in OAS.

\section{Future Directions in Service Delivery}

Popuri Krishna, Shabbir Amanullah, Monica Bretzlaff

Background: With the increasing service needs of our senior with Dementia specially trained services are needed to help families and the frontline care providers to manage the responsive behariors due to dementia. Behavior Support specialist teams are organised in Ontario to meet this challenge. The symposium will critically evaluate the benefits and the gaps with this system and propose innovative service delivery model to improve the Resilience of care providers.

Method: examine the existing systems in the community in ontario for senior care.

Results: evaluate the potential gaps for the service needs.

Conclusions: alternative care models are needed for future care needs of this challenging population.

Outcomes of a Novel Educational Intervention: Teaching Junior Psychiatry Residents Capacity Assessment with Simulated Patients

Ian Mackay

Background: The use of simulated patients (SPs) in medical education has been employed since 1964. SP use has permeated most medical schools, with research supporting SPs in many aspects of medical training. A literature search suggests SPs have not been used to help psychiatry residents learn how to expertly assess the capacity of patients and medical colleagues often look to psychiatry when complex patient capacity issues arise. At Dalhousie, there is no formal teaching on capacity assessment; learning for junior residents depends on clinical chance. Assessment of capacity using simulated patients might create more reliable, safe and enjoyable learning environments for junior psychiatry residents.

Method: This curriculum was informed by an existing resource used to introduce capacity assessment to Dalhousie medical students. The SP case was augmented for 10-16 PGY1 and 2 psychiatry residents to be given March, 2019 over two hours. A didactic session precedes dividing the group into three. Small groups will assess the SP for one of: capacity to consent to treatment, financial, or personal care capacity. Another SP, playing the son, will be available for collateral information. The groups will present their conclusions, then five brief capacity case scenarios will be discussed. A post-session survey using the Likert scale will be given to the residents gauging change in comfort level with capacity assessment and seeking feedback on format and content to guide future use.

Results: The educational intervention was well received. $100 \%$ of residents present indicated it was "very good", "excellent", or "outstanding". A total of $60 \%$ of the participants completely agreed using the Simulated Patient was important to the effectiveness of the session, while another $30 \%$ somewhat agreed. The intervention increased confidence in assessing capacity in $90 \%$ of those attending the session. Potential barriers identified included the amount of time for preparation of the teaching material as well as access to, training of and cost associated with the simulated patients.

Conclusions: This novel educational intervention to teach junior psychiatry residents capacity assessment with simulated patients was a success. This success was despite the small sample size, and several potential barriers to implementing this session into the core psychiatry resident curriculum. Next steps include incorporating feedback to improve future sessions and assessing learning with pre- and post testing, and ultimately determining if this intervention changes practice.

\section{Autoimmune Encephalitis in Older Adults: A Review of Clinical Features, Treatment, Outcomes and Future Directions}

Joseph Emerson Marinas, Dmitriy Matveychuk, Serdar M. Dursun, Glen B. Baker

Background: In recent years, there has been markedly increased interest in autoimmune encephalitides a group of conditions with neuropsychiatric presentations. Of these conditions, anti-N-methyl-D-aspartate (NMDA) receptor encephalitis has been the most extensively studied and it serves as a model for investigating pathological mechanisms and potential treatments for antibody-mediated encephalitides. There are limited studies, however, regarding the clinical presentation and outcomes of autoimmune encephalitis in the geriatric population. Given the development of changes in mood, anxiety, and cognitive dysfunction that occur with autoimmune encephalitides, these conditions may be considered when investigating neurocognitive disorders.

Method: We searched the databases PubMed, Web of Science, and PsycINFO with the search terms "autoimmune 


\section{CAGP-CCSMH ABSTRACTS}

encephalitis" and "antibody-mediated encephalitis" in combination with the terms "older adults" and "geriatrics" for literature published between the years 2000 and 2018 inclusive. We also searched the references of the articles reviewed to investigate clinical features, outcomes, and prognosis of autoimmune encephalitides.

Results: The literature regarding autoimmune encephalitides in adults 65 years of age and older is limited to groups within case series. Older adults generally have longer times prior to diagnosis, decreased frequency of teratoma but increased likelihood of malignancy, greater likelihood of presenting with memory deficits, and greater residual symptom burden in comparison to younger adults.

Conclusions: Autoimmune encephalitis remains a challenging diagnosis in the geriatric population. Medical comorbidities and frailty may complicate diagnosis and treatment strategies with older adults. Given the limited body of research, further investigation may help develop approaches tailored to geriatric patients with autoimmune encephalitis.

The Association between Quality Indicators and Inspection Performance in Long-Term Care in Ontario: A Machine Learning Study

Pouria Mashouri, Babak Taati, Andrea Iaboni

Background: 9 quality indicators are publicly reported to allow an evaluation of quality in long-term care (LTC) facilities within Ontario. Separately and independently, inspections are conducted by the Ministry of Health and Long-Term Care of Ontario (MOHLTC), to identify failure to comply with LTC legislation or regulations. In this study, we aimed to examine the relationship between quality indicators and compliance in LTC facilities across Ontario.

Method: We employed machine learning techniques to examine if quality indicators can be used to classify each home into three categories (in good standing, needing improvement, needing significant improvement) based on inspection results. A simple linear model was purposefully chosen to allow for feature analysis.

Results: After running a wide-range of models, we found only a weak relationship between quality indicators and compliance. The best-performing model was only able to achieve an accuracy of $40.1 \%$. Feature analysis was then performed on the final model to identify which quality indicators were most indicative of compliance. 'Experiencing Worsened Pain', Restraint Use', and 'Worsened Pressure Ulcers' were strongly correlated with homes "needing significant improvement." Counter-intuitively, 'Improved Physical Functioning' had an inverse relationship with homes "in good standing."
Conclusions: Overall, this study can be seen as a first step towards empirically assessing the different methods that currently exist to measure quality of care in LTC facilities in Ontario. Further work is required to understand the limited relationship between these two datasets, as well as attempting to identify those quality indicators that most meaningfully represent quality of care.

\section{Delirium in Geriatric Population}

Jordan McNeil

Background: Using the 3D's Best Practice Guidelines (Delirium, Dementia and Depression) our organization is implementing the Confusion Assessment Model to our patient assessments as well as new documentation methods to guide the care of patients who are screened as delirious.

Method: The Confusion Assessment Model (CAM) is used on all patients over aged 65 to screen for early detection of delirium. Nurses who perform the CAM screening and detect a patient to be delirious use a clinical pathway which is specific to delirium risk factors. This allows for a thorough documentation strategy to aid the nurse to identify the underlying source(s) or cause of delirium.

Results: Nurses have been educated on how to perform the CAM assessment and how to use the delirium clinical pathway to identify contributing factors to a patients delirium. The RNAO best practice guidelines for delirium provide strategies for non-pharmacological interventions and a person centered care plan. Interventions planning and evaluation recommendations are all from the best practice guideline.

Conclusions: Raising awareness to the validity of the best practice guidelines is important in supporting the needs of patients at risk for delirium. Detecting risk factors for delirium is a primary prevention method which aims to identify vulnerable persons sooner rather than later. Delirium education and evidenced based recommendations help to empower and support front line health care workers, patients and their families.

\section{Prevalence of Apathy in Parkinson's Disease: An Updated Systematic Review and Meta-analysis}

Bria Mele, Daria Merrikh, Zahra Goodarzi

Background: Apathy in Parkinson's disease (PD), has a reported prevalence of $40 \%$. This estimate comes from a systematic review and meta-analysis published in 2015. It has bene reported that $47 \%$ of all literature on apathy in PD was published after 2014, thus an updated systematic review to better understand prevalence is required. It was the aim of 
this research to conduct an updated systematic review on the prevalence of apathy in PD populations.

Method: Six medical databases were searched, including MEDLINE, EMBASE, PsycINFO, CINAHL, the Cochrane Database of Systematic Reviews, and the Cochrane Central Register of Control Trials. The main search terms were "Parkinson's disease" and "Apathy", combined using Boolean operators as appropriate. Pooled estimates, heterogeneity, and $95 \%$ confidence intervals were calculated using a randomeffects model. The Mantel-Haenszel-weighted DerSimonian and Laird Models were used to calculate prevalence.

Results: Five detection tools were used to identify apathy; including the Apathy Scale, the Lille Apathy Rating Scale, the Apathy Evaluation Scale, the Unified Parkinson's Disease Rating Scale Part I, and the Neuropsychological Inventory. The pooled prevalence for the updated 19 unique study populations was $23.9 \%$ (95\% CI $17.3 \%-30.4 \%)$. There was high, statistically-significant heterogeneity associated with this value ( $\mathrm{I} 2=95.4 \% ; \mathrm{p}=0.00$ ).

Conclusions: The updated population of apathy in PD populations in $24 \%$. There is a large amount of heterogeneity associated with this value, thus it is suggested future research must focus on consistency in detection tools for apathy across PD populations.

Evaluation of Anticonvulsants Use in Management of Alcohol Withdrawal Syndrome and Long-term Medication Management of Alcohol Use Disorder in Older Adults

Stefanie Montgomery, Gillian Wang, Karen Dahri, Peter Chan

Background: Alcohol withdrawal syndrome (AWS) occurs after discontinuation of excessive alcohol consumption. The geriatric population is at higher risk for AWS complications due to a greater prevalence of comorbidities, a longer duration of alcohol abuse, baseline cognitive deficits, and sensitivity to treatment. Benzodiazepines remain the mainstay therapy of seizures and delirium tremens. The Prediction of Alcohol Withdrawal Severity Scale (PAWSS), validated in the medically ill, can be a useful tool for identifying those at moderate or high risk for AWS so that benzodiazepine and adjunctive treatments, such as anticonvulsants may be considered. Vancouver General Hospital (VGH) has pre-printed order sets that follow the Clinical Institute Withdrawal Assessment-Alcohol revised (CIWA-Ar) format to provide symptom-triggered benzodiazepine therapy. The use of anticonvulsants in the elderly population for alcohol withdrawal has limited evidence despite being potentially efficacious and safe while providing mood stabilizing or anxiolytic effects. Inpatient management often focuses on the withdrawal symptoms and it is unclear how much attention is given to the long-term treatment of alcohol use disorder using agents such as disulfiram, naltrexone and acamprosate.
Method: We are conducting a retrospective chart review of 216 patients aged 60 and over admitted to medicine units that are started on the CIWA-Ar protocol between June 2013 - June 2018. The primary objectives are whether adjunctive anticonvulsants for AWS or long-term treatments for alcohol use disorder are initiated. Secondary objectives include adverse events secondary to the treatment of anticonvulsants and benzodiazepine therapy and a quality assurance of the CIWA-Ar protocol.

Results: Pending

Conclusions: Pending

Design and Evaluation of an Ecological Momentary Assessment Tool for Assessing Depression in Dementia

Iulia Niculescu

Background: Assessing depression in people with dementia is challenging due to limitations of retrospectivity. Mobile Ecological Momentary Assessment (EMA) provides a novel approach in assessing depressive symptoms by collecting informant measures in intervals throughout the day, decreasing recall bias and increasing representativeness.

Method: The objective of this study is to design an EMA application for assessing depression in individuals with dementia. A literature review was conducted to determine commonly used and validated assessments for depression in dementia. Assessments were analyzed for common items, where items less commonly used ( $<50 \%$ of assessments sharing the item) or not relevant to be collected using EMA were excluded. Wording of items were also analyzed to develop the specific structure of questions for the EMA assessment.

Results: Six assessments were found and demonstrated adequate performance outcomes. Items fell into either moodrelated, dementia-related, vegetative, psychotic or positive mood symptom groups. The mood-related group was analyzed separately for prominent items, which were sadness, anxiety, pessimism, loss of interest and irritability. Wording of items were modified to be consistent with being collected throughout the day, rather than retrospectively. These items were incorporated as core observational domains in the application to be tested. Sadness and anxiety were additionally included as self-report items as previous studies have shown these to be most discordant between individuals with dementia and informants.

Conclusions: This research is a first step towards an innovative approach to assessing depression in dementia. Next steps involve an evaluation of the application's feasibility and reliability for assessing depression in individuals with dementia. 
Screening for Depression in People with Cognitive Impairment Receiving Home Care: A Systematic Review

Iulia Niculescu, Twinkle Arora, Andrea Iaboni

Background: Previous systematic reviews have investigated the performance of depression screening tools in older adults with cognitive impairment $(\mathrm{CI})$ in clinics, nursing homes and residential care. Less is known about depression screening in home care, which is important to consider because the prevalence of CI and depression is high in home care.

Method: The objective of this study is to identify the best performing, evidence-based depression screening tool for individuals with CI receiving home care by first assessing the performance of depression screening tools and second, establishing the current evidence for depression screening practices. A systematic search was conducted using MEDLINE, EMBASE, Health and Psychosocial Abstracts, PsycINFO and CINAHL with the following criteria: a sample of older adults ( $>55$ years) with CI receiving home care, where performance outcomes of the assessed depression screening tool are reported. Data extraction and risk of bias assessments were completed.

Results: Of 5736 studies, three passed eligibility criteria: The Patient Health Questionnaire $(\mathrm{n}=37)$, the Geriatric Depression Scale $(n=49)$ and the Mental Health Index $(n=396)$ were assessed in samples of individuals with and without CI. Performance outcomes demonstrated low success in subsamples of CI. Furthermore, all studies showed varying degrees of risk of bias across categories of patient selection, study flow and timing, and use of reference standard and screening tools.

Conclusions: Little evidence can be concluded concerning the best screening practices among recipients of home care with CI from current studies. Limitations include biases in study methods and a general lack of existing studies.

\section{The Impact of Music Therapy: Quality of Life Measures and BPSD in Longterm Care}

Donovan Nolan, Alexandra Moir, Paige Morrissey, Amy Gough, Keri-Leigh Cassidy

Background: Neuropsychiatric symptoms (NPS) of dementia present some of the most challenging aspects of dementia care in the long-term care setting. Music therapy was introduced in two long term care facilities in Nova Scotia as a nonpharmacological intervention to treat the NPS of dementia. Music therapy has been shown to reduce challenging behaviors associated with cognitive impairment and this project also wanted to assess music's impact on resident satisfaction ratings, mood symptoms, pre and post incidence of BPSD and facility satisfaction ratings before and after initiating the music therapy intervention.

Method: Using an observational within-subject control design, the impact of half-time music therapy on residents was evaluated in 16 residents with NPS of dementia as measured on the Cohen-Mansfield Agitation Inventory over a 12-month period, and resident quality of life was measured on the Cornell-Brown Scale for Quality of Life in Dementia. The 16 participants ranged from age 76 to 92 , with moderate to severe dementia (average MMSE 9.53).

Results: Monthly Cornell-Brown scores demonstrated significant improvement in 7 out of 12 months they received treatment, relative to a pre-intervention baseline and low levels of agitation were seen in residents during active treatment. Staff perception of the impact of music therapy on the overall long term care environment was assessed using a quality assurance survey, and showed a perceived improvement in both morale and resident behaviours.

Conclusions: The results support the inclusion of music therapy in long term care for dementia even at the level of one half-time position.

\section{Simple Schizophrenia in 65-Year-Old Neglected Women}

Santosh Kumar Pandit

Background: Mental health is very neglected in all most all continent and more in asian and especially developing countries. Neglect is more prominent toward feminine. This case is a live example of that and the women suffered almost 25 yeats without treatment. Lost her most fertile life as a victim of insanity just because no one from her family dare to bring her to psychiatrist but took her to holli healer many time. Her life was fully disabled by insanity till she got recognised by my team and got treatment. Now she is doing well on antipsychotic treatment.

Method: Demographic data is collected from reliable and adequate informant and a good quality of history with multiple time application of prognostic scales. M.S.E. done repetitively and comparison with other possible Differential diagnosis is done before reaching to final diagnosis.

Results: This is a case of simple schizophrenia from last 25 years in a neglected 65 years old women on exclusion of all other possible D.D.

Conclusions: Simple schizophrenia is treatable if negligence from the family and social stigma is obliterated and life could be cheerful for women if feminine legligency is mitigated and a social support and stigma free unbiased, nonjudgmental approach is welcomed towards the mental health of old women. 


\section{Pilot Randomized Control Trial Examining Tai-Chi/Qi- gong in Older Adults with Bipolar Disorder}

Haley Park, Soham Rej, Suzanne Renaud, Zoe Thomas, Lisa Eyler, Serge Beaulieu

Background: It is expected that by 2030, the percentage of patients with bipolar disorder over the age of sixty will exceed $50 \%$. Older adults with bipolar disorder (OABD) particularly suffer from poor cognition and persistent sub-syndromal depressive symptoms that impede daily functioning, for which current pharmacotherapy is often limited and poorly tolerated with unwanted side effects. As such, alternative treatment options are urgently needed. Mind-body interventions have recently been evidenced to be effective in treating various psychiatric illnesses. Tai-chi/qigong may be an especially appropriate form of moving mindfulness for OABD due to the accessibility of gentle physical movements.

Method: We will conduct a 12-week two-arm pilot randomized control trial (RCT) comparing $1 \mathrm{~h} /$ week of Taichi/Qigong (treatment) vs. walking (active control) in 30 euthymic (Montgomery-Asberg Depression Rating Scale (MADRS) and Young Mania Rating Scale < 10) OABD patients (age $x x>50$ years). We will measure depressive symptoms (MADRS) and cognition (International Society for Bipolar Disorders-Battery for Assessment of Neurocognition) at Baseline, 12-weeks, and 24-weeks follow-up, with the primary outcome being reduction in depressive symptoms or prevention of relapse, and the secondary outcome being improvement in cognition.

Results: The study protocol will be presented at the CAGP Conference in Banff, October 2019. Results will be ready by Fall 2020. Our methods and results may involve fMRI analysis upon pending reception of funding.

Conclusions: Tai-chi/Qigong presents a cost-effective, scalable, and tolerable treatment option to improve depression and cognition in OABD. This pilot study will provide preliminary data for a larger RCT.

Pragmatic Education RCT: Early Clinical Exposure to Geriatric Psychiatry and Medical Students' Interest in Caring for Older Adults

Klara Pokrzywko, Susana Gabriela Torres-Platas, Petal Abdool, Marouane Nassim, Trent Semeniuk, Tricia Woo, Marie-Andrée Bruneau, Tarek Rajji, Soham Rej

Background: In the next 25 years, the population aged 65+ will nearly double in many countries, with few new doctors wishing to care for older adults. We hypothesize that early clinical exposure to elderly patients' care could increase students' interest in caring for older adults during their future career.

Method: We conducted a pragmatic medical education randomized controlled trial at the Jewish General Hospital and the Douglas Mental Health Institute, McGill University in Montreal, Canada. 3rd year Medical students undergoing their mandatory 16-week half-time clerkship rotation in psychiatry were randomized to the equivalent of 2-4 weeks' full-time exposure to clinical geriatric psychiatry $(\mathrm{n}=84)$.

Results: Being randomized to geriatric psychiatry exposure $(n=44 / 84)$ was associated with increased "comfort in working with geriatric patients and their families" at 16-week followup $(59.1 \%$ vs. $37.5 \%, \chi 2(1)=3.9, p=0.05)$. However, there was no significant association found between geriatric psychiatry exposure and change "in interest in caring for older adults" or change in "interest in becoming a geriatric psychiatrist".

Conclusions: The results of this pragmatic education RCT suggest that exposing 3rd year medical students to 2-4 weeks of geriatric psychiatry did not increase their interest to care for older adults or become a geriatric psychiatrist. However, it did increase their comfort level in working with older adults and their families. However, more research is necessary to identify potential interventions that could inspire and increase medical students' interest in caring for older adults as part of their future careers.

Clinical Profile of Older Alternate Level of Care (ALC) Adults with Mental Illness Discharged to a Reintegration Unit (RIU) from Acute Care

Aviva Rostas, Debra Walko, Melonie Hopkins, Nicole Downes, Lindor Uygur, Scott McKay

Background: White Squirrel Way (WSW) is a new and unique RIU designed to facilitate transition from ALC beds into the community and to relieve acute care bed pressures at the Centre for Addiction and Mental Health (CAMH). It is a partnership between LOFT Community Services and CAMH and has the services of a geriatric psychiatrist to support clients in the program and collaborate with LOFT staff. This study aims to describe demographics, clinical variables, and transition details among individuals discharged to WSW since its inception in December 2017.

Method: A retrospective, observational study is planned of patients discharged to WSW since its inception in December 2017. Current WSW residents will be interviewed regarding their personal experiences as ALC clients.

Results: pending

Conclusions: Understanding the clinical profiles, transition details, experience of the transition, and outcomes of WSW 


\section{CAGP-CCSMH ABSTRACTS}

residents will lead to increased knowledge about this complex and difficult to discharge population. This can help to reduce barriers to successful transitions.

Explorer les besoins des proches aidants dans la prise en charge des patients ayant des symptômes comportementaux et psychologiques de la démence (SCPD) en centre d'hébergement et de soins de longue durée (CHSLD)

Jessika Roy Desruisseaux, Sarah Pham, Myriam BélangerDibblee, Hubert Tremblay

Background: La grande majorité des patients atteints de troubles neurocognitifs développent des symptômes comportementaux et psychologiques de la démence (SCPD). Ces comportements perturbateurs sont généralement le reflet de besoins non comblés. Les SCPD peuvent significativement entraver la qualité des soins dont bénéficient les patients en hébergement. Les experts reconnaissent que les approches non pharmacologiques sont à prioriser comparativement aux approches pharmacologiques. En assurant souvent une grande part des soins aux personnes âgées en perte d'autonomie, les proches aidants sont susceptibles d'avoir développé une approche adaptée aux patients et sont ainsi une source significative d'informations. Or, la transition en CHSLD implique un changement de rôle du proche aidant qui n'est pas toujours impliqué au sein de l'équipe médicale. Cette transition représente aussi une grande période d'adaptation pour le patient. Cette étude explore donc les besoins des proches aidants lorsque le patient est en CHSLD et, l'impact de ces besoins non comblés.

Method: Il s'agit d'un projet de recherche qualitatif. L'expérience des proches aidants de patients atteints de troubles neurocognitifs résidant dans un CHSLD est explorée à travers des entrevues de groupe semi-structurées. Ces entrevues ont été conduites jusqu'à saturation des données dans les entretiens. Le contenu des entrevues a ensuite été analysé afin d'en dégager le vécu et les besoins des proches aidants

Results: Les résultats préliminaires de l'analyse seront présentés au congrès.

Conclusions: À venir.

\section{Detection of Anxiety in Older Adults: A Systematic Review and Meta-Analysis}

Leyla Samii, Michael Walsh, Jayna Holroyd-Leduc, Zahra Goodarzi

Background: Anxiety disorders are common mental health disorders in elderly that are associated with poor health outcomes. In outpatient setting, physicians need accurate diagnostic tools to enable them to detect anxiety disorders in the elderly population. Our objective was to identify the anxiety detection tools in the elderly and to find which tools were the most accurate in detecting anxiety in comparison with gold standard.

Method: We conducted a systematic review of MEDLINE, EMBASE, PsycINFO, the Cochrane databases, and the grey literature. We included diagnostic accuracy studies that compared an anxiety detection tool with gold standard of DSM or ICD in participants over the age of 50 in outpatient setting with anxiety related illnesses as defined by gold standard. Abstracts and full texts were reviewed by two authors. The proposal was registered with PROSPERO CRD42016051620 and review follows PRISMA reporting criteria.

Results: We identified 10417 abstracts and 1137 articles were reviewed at the full text stage. Articles that compared the accuracy of the diagnostic tools with gold standard were selected for data extraction.

Conclusions: There are many available tools for the detection of anxiety in seniors. Clinical practitioners need to be aware of breadth of available tools and their associated accuracy such that clinical decisions are informed by the most accurate, but also feasible tools.

\section{Mental Health and Cognition in Older Cannabis Users: A Review}

Blanca Elena Vacaflor, Olivier Beauchet, G. Eric Jarvis, Alessandra Schavietto, Soham Rej

Background: The impact of cannabis use on mental health and cognition in older adults is unclear. This review summarizes the literature examining: 1) mental health and cognitive correlates of cannabis use in older adults from epidemiological studies, and 2) mental health and cognitive adverse effects of medical cannabis initiation in older adults.

Method: Medline was searched for articles describing 1) mental health and cognitive comorbidities of cannabis use and 2) mental health and cognitive adverse effects of medical cannabis use, in adults aged $50+$.

Results: A total of 17 relevant studies were identified, including nine cross-sectional studies on mental health comorbidities of older cannabis users. The prevalence of mental and substance use disorders is approximately two to three times higher in older adults who have used cannabis in the past year, compared to older adults who used more than a year ago and those who never used. Cognition was not assessed in those studies. The remaining eight studies - half of these being small clinical trials with $\mathrm{n}<50$ - found that short term, low dose medical cannabis was generally well tolerated in older 
adults without prior serious mental illness. However, mental/ cognitive health was not systematically assessed.

Conclusions: Older cannabis users have higher rates of mental health and substance use disorders, highlighting a potential need for more screening and treatment of mental health disorders in this population. Larger studies on the safety of medical cannabis in older adults using standardized cognitive and mental health assessments are needed.

\section{What Are the Factors Associated with Self-Reported Health in Community-Dwelling Older Adults?: A Scop- ing Review}

Carly Whitmore, Maureen Markle-Reid, Carrie McAiney, Kathryn Fisher, Jenny Ploeg

Background: Self-reported health is a subjective assessment of health based upon individual perceptions. It is a widely used measure of health in epidemiological studies because of its association with morbidity and mortality - especially among older adults. Despite being widely collected in health surveys and utilized in research, there remains a need to better understand what physical, psychosocial, individual- and community-level factors are associated with self-reported health among older adults. The aim of this review was to articulate the factors associated with self-reported health among community-dwelling older adults.

Method: A scoping review was conducted. Four hundred and thirty-one manuscripts were identified from MEDLINE, CINAHL, Web of Science, and AgeLine. Eighteen additional manuscripts were identified through hand searching. Manuscripts were screened for relevancy and data were abstracted independently by two reviewers.

Results: Thirty quantitative studies were reviewed. Designs included cross-sectional, longitudinal, and meta-analysis. Four categories, representing 52 individual factors, were found to be associated with self-reported health among community-dwelling older adults. These categories included: sociodemographic; physical and psychiatric health; healthrelated behaviour; and emotional factors. The findings suggest that the presence of multiple chronic conditions, specifically, the presence of depressive symptom among older adults, is a significant factor that impacts self-reported health. There is a need to further explore the impact of depressive symptoms and depression on self-reported health among this population.

Conclusions: Factors associated with self-reported health among community-dwelling older adults are wide-ranging across three broad groups. Further research is warranted to examine how depressive symptoms and depression influence self-reported health in this population.

\section{Designing a Qualitative Study on the Transition from Specialized Psychogeriatric Dementia Units to Long- Term Care}

Song Yang Yu, Andrea Iaboni

Background: The past several decades have seen the emergence of specialized psychogeriatric dementia units (SPDUs) in tertiary care as an important setting for the management of severe behavioural and psychological symptoms of dementia (BPSD). There is a paucity of literature examining the transition from SPDU back to long-term care (LTC), once sustained improvement in BPSD is achieved.

Method: A literature review was conducted to determine the factors that are involved in successful treatment on an SPDU, as well as factors that impact the transition from SPDUs to LTC. This information was used to design a qualitative study that explores the views and priorities that families and LTC staff have on the transition from SPDUs to LTCs.

Results: Compared to LTC, SPDUs typically involve higher staff to patient ratios and greater emphasis on nonpharmacological interventions. SPDUs have been shown to be effective in reducing the severity of BPSD, although they do not impact the rate of cognitive and functional decline. Family engagement is important to the success of interventions on an SPDU. Based on the literature review, we have designed a qualitative research study to help understand barriers and facilitators to transition to LTC after a stay on an SPDU.

Conclusions: This literature review and protocol design is the first step towards conducting a qualitative study on the transition from SPDU to LTC. Next steps involve ongoing data gathering and analysis, in coordination with the SPDU at Toronto Rehabilitation Institute and local LTC facilities.

\section{Association of Intensive Hypertension Control with Mild Cognitive Impairment and Dementia in Older Adults with Depression}

Anthony Yeung, Damien Gallagher

Background: There is a long-established association between hypertension and the increased risk of age-related cognitive decline and dementia. However, the direct effects of antihypertensive treatment and whether it reduces the risk of dementia is less clear. SPRINT (Systolic Blood Pressure Intervention Trial) MIND, a substudy of the controversial landmark trial, revealed that aggressive lowering of blood pressure $(<120$ $\mathrm{mmHg}$ systolic) did not reduce dementia risk. Despite the high comorbidity between depression and hypertension, the role of intensive hypertension control in older adults with depression is not well understood. In addition, depression 


\section{CAGP-CCSMH ABSTRACTS}

is increasingly recognized as an independent risk factor for cognitive impairment and dementia. Therefore, we sought to: (1) describe the overall risk of progression to Alzheimer's dementia (AD) among a large population of hypertensive older adults attending specialized memory services and to (2) identify whether intensive hypertension treatment would decrease the risk of progression to $\mathrm{AD}$ in both depressed and non-depressed individuals.

Method: Using data from the National Alzheimer's Coordinating Centre, 4,105 study participants with normal cognition and clinically defined hypertension were followed until development of MCI or AD. Subanalyses included comparing outcomes between depressed and non-depressed participants. Multivariate survival analyses were also performed to compare outcomes between 3 different hypertension treatment groups (1) < $120 \mathrm{mmHg}$ systolic, (2) 121-139 mm Hg systolic, and (3) $140 \mathrm{mmHg}$ systolic.

Results: To be presented at meeting.

Conclusions: To be presented at meeting. 